\title{
Water ice deuteration: a tracer of the chemical history of protostars ${ }^{\star}$
}

\author{
V. Taquet ${ }^{1}$, P. S. Peters ${ }^{1,2}$, C. Kahane ${ }^{1}$, C. Ceccarelli ${ }^{1}$, A. López-Sepulcre ${ }^{1}$, C. Toubin ${ }^{2}$, D. Duflot ${ }^{2}$, and L. Wiesenfeld ${ }^{1}$ \\ 1 UJF-Grenoble 1/CNRS-INSU, Institut de Planétologie et d'Astrophysique de Grenoble (IPAG) UMR 5274, 38041 Grenoble, France \\ e-mail: vianney.taquet@obs.ujf-grenoble.fr \\ 2 Laboratoire de Physique des Lasers, Atomes et Molecules (PhLAM), UMR CNRS 8523, Universite Lille 1, \\ 59655 Villeneuve d'Ascq Cedex, France
}

Received 23 July 2012 / Accepted 5 November 2012

\begin{abstract}
Context. Millimetric observations have measured high degrees of molecular deuteration in several species seen around low-mass protostars. The Herschel Space Telescope, launched in 2009, is now providing new measures of the deuterium fractionation of water, the main constituent of interstellar ices.

Aims. We aim at theoretically studying the formation and the deuteration of water, which is believed to be formed on interstellar grain surfaces in molecular clouds.

Methods. We used our gas-grain astrochemical model GRAINOBLE, which considers the multilayer formation of interstellar ices. We varied several input parameters to study their impact on water deuteration. We included the treatment of ortho- and para-states of key species, including $\mathrm{H}_{2}$, which affects the deuterium fractionation of all molecules. The model also includes relevant laboratory and theoretical works on the water formation and deuteration on grain surfaces. In particular, we computed the transmission probabilities of surface reactions using the Eckart model, and we considered ice photodissociation following molecular dynamics simulations.

Results. The use of a multilayer approach allowed us to study the influence of various parameters on the abundance and the deuteration of water. Deuteration of water is found to be very sensitive to the ortho-to-para ratio of $\mathrm{H}_{2}$ and to the total density, but it also depends on the gas/grain temperatures and the visual extinction of the cloud. Since the deuteration is very sensitive to the physical conditions, the comparison with sub-millimetric observation towards the low-mass protostar IRAS 16293 allows us to suggest that water ice is formed together with $\mathrm{CO}_{2}$ in molecular clouds with limited density, whilst formaldehyde and methanol are mainly formed in a later phase, where the condensation becomes denser and colder.
\end{abstract}

Key words. astrochemistry - ISM: abundances - ISM: clouds - ISM: molecules - molecular processes - stars: formation

\section{Introduction}

Understanding the formation of water is crucial, not only because of its primordial importance for life on Earth, but also because it is thought to be one of the most abundant oxygenbearing species and also one of the main gas coolants (Ceccarelli et al. 1996; Kaufman \& Neufeld 1996; van Dishoeck et al. 2011). Interstellar water is believed to be formed mainly via three main mechanisms: 1) cold gas-phase chemistry, starting from the ionization of $\mathrm{H}_{2}$ by cosmic rays, eventually leading to $\mathrm{H}_{3} \mathrm{O}^{+}$via ion-neutral reactions that then recombine with electrons to form $\mathrm{H}_{2} \mathrm{O}$ (Bates 1986; Hollenbach et al. 2009); 2) on the surface of interstellar dust particles, via the hydrogenation of accreted atomic and molecular oxygen occurring at cold temperatures (Tielens \& Hagen 1982; Cuppen \& Herbst 2007; Miyauchi et al. 2008); 3) warm gas chemistry, initiated by a few endothermic reactions involving $\mathrm{H}_{2}$ in warm gas $(T>250 \mathrm{~K})$ (Ceccarelli et al. 1996; Kaufman \& Neufeld 1996).

The advent of space telescopes, combined with groundbased observatories, has allowed astronomers to observe vapours and ices of water in several phases of star formation. Water vapour is present in cold molecular clouds and prestellar cores

* Appendices are available in electronic form at http: //wwW . aanda.org but only with low abundances $\left(X_{\text {gas }}\left(\mathrm{H}_{2} \mathrm{O}\right) \sim 10^{-8}-10^{-9}\right.$ relative to $\mathrm{H}$ nuclei, see Bergin \& Snell 2002; Klotz et al. 2008; Caselli et al. 2010). In cold clouds, water is likely condensed in ices $\left(X_{\text {ice }}\left(\mathrm{H}_{2} \mathrm{O}\right) \sim 5 \times 10^{-5}-10^{-4}\right.$, Whittet \& Duley 1991; Pontoppidan et al. 2004). Hot corinos and outflows of low-mass Class 0 protostars show higher abundances of gas phase water, with abundances of a few $10^{-6}$ in hot corinos (Ceccarelli et al. 2000; Coutens et al. 2012; Kristensen et al. 2012), whilst protostar outflows show higher abundances up to a few $10^{-5}$ (Liseau et al. 1996; Lefloch et al. 2010; Kristensen et al. 2010, 2012). Water ice has also been observed towards cold protostellar envelopes with similar abundances to molecular clouds $\left(\sim 10^{-4}\right.$, Pontoppidan et al. 2004; Boogert et al. 2008). Recent infrared observations have also shown the presence of water in protoplanetary disks in different states: water ice (Terada et al. 2007) and hot and cold water vapour (Carr \& Najita 2008; Hogerheijde et al. 2011, with abundances of $10^{-4}$ and lower than $10^{-7}$, respectively). Analysis of debris disks show that dust particles are covered by ice mixtures (e.g. Li \& Greenberg 1998; Lebreton et al. 2012).

To summarise, it is now accepted that water is present during all phases of the star formation process. However, its evolution from molecular cloud to planetary system still remains unclear. The deuterium fractionation can help us constrain its formation and its evolution. First, it can probe the formation pathways of 
water observed in the early stages because of its sensitivity to the physical conditions. Second, it allows us to investigate its reprocessing in protoplanetary disks, and eventually to determine whether water on Earth has an interstellar origin. Comparing the $\mathrm{HDO} / \mathrm{H}_{2} \mathrm{O}$ ratio in comets and Earth is, for example, important for evaluating the possible contribution of comets for transferring water in Earth's oceans (Owen \& Bar-Nun 1995). Recent Herschel observations have reported a $\mathrm{D} / \mathrm{H}$ ratio of water $(0.014 \%)$ in the Jupiter family comet $103 \mathrm{P} /$ Hartley 2 originating in the Kuiper belt, very similar to the value for the Earth's oceans, supporting the hypothesis that a part of water comes from comets (Hartogh et al. 2011).

The $\mathrm{HDO} / \mathrm{H}_{2} \mathrm{O}$ ratio has recently been evaluated in the gas phase of low-mass Class 0 protostar envelopes with values varying from less than $10^{-4}$ in NGC 1333-IRAS 4B (Jørgensen \& van Dishoeck 2010) to more than $10^{-2}$ in NGC 1333-IRAS 2A (Liu et al. 2011). The low-mass Class 0 protostar IRAS 162932422 seems to have the most reliable value since the main isotopologue (via $\mathrm{H}_{2}^{18} \mathrm{O}$ ) and its simply and doubly deuterated isotopologues have been observed several times via ground-based and space telescopes (Ceccarelli et al. 2000; Parise et al. 2005; Butner et al. 2007; Vastel et al. 2010; Coutens et al. 2012). The most recent work by Coutens et al. (2012) reports an $\mathrm{HDO} / \mathrm{H}_{2} \mathrm{O}$ ratio of $\sim 3 \%$ in the hot corino, $\sim 0.5 \%$ in the cold envelope, and $\sim 5 \%$ in the photodesorption layer of the foreground cloud. Observations of $\mathrm{D}_{2} \mathrm{O}$ by Butner et al. (2007) and Vastel et al. (2010) give a $\mathrm{D}_{2} \mathrm{O} / \mathrm{H}_{2} \mathrm{O}$ ratio of $\sim 10^{-3}$. Using the $4.1 \mu \mathrm{m}$ OD stretch band, solid HDO has been observed towards a sample of low- and high-mass stars by Dartois et al. (2003) and Parise et al. (2003). These observations provide an upper limit for the solid abundance ratio between 0.2 and $2 \%$. Although these values are much higher than the cosmic elemental abundance of deuterium $\left(1.5 \times 10^{-5}\right.$ Linsky 2003$)$, water seems to be less deuterated than the other molecules also mainly formed on grain surfaces, such as formaldehyde and methanol. Figure 1 graphically shows that water possesses a lower level of deuteration, with a mean $\mathrm{HDO} / \mathrm{H}_{2} \mathrm{O}$ ratio of $\sim 3 \%$, whereas other molecules have a fractionation of their singly deuterated isotopologue higher than $10 \%$.

$\mathrm{H}_{2} \mathrm{O}$, but also $\mathrm{H}_{2} \mathrm{~S}, \mathrm{H}_{2} \mathrm{CO}$ and $\mathrm{CH}_{3} \mathrm{OH}$ (and $\mathrm{HCOOCH}_{3}$ ) are thought to be mainly formed on grain surfaces via the hydrogenation of accreted $\mathrm{O}\left(\right.$ or $\left.\mathrm{O}_{2}\right), \mathrm{S}$, and $\mathrm{CO}$ from the gas phase. Cold gas-phase chemistry produces water vapour with low abundances (a few 10 ${ }^{-7}$; Bergin et al. 2000), whilst endothermic reactions producing warm water are efficient at $T>250 \mathrm{~K}$. In contrast, water ice desorbs into the gas phase in the envelope of Class 0 protostars in large quantities at $T \sim 100 \mathrm{~K}$. Therefore, the deuteration of water measured by millimetric observations likely reflects the deuteration of its icy precursor governed by the accretion of gas phase $\mathrm{H}$ and $\mathrm{D}$ atoms. Indeed, the timescale needed to significantly alter the deuteration after evaporation in warm gas is longer than the typical age of Class 0 protostars $\left(\sim 10^{5}\right.$ versus $\sim 10^{4} \mathrm{yr}$, Charnley et al. 1997; Andre et al. 2000). Roberts et al. (2003) showed that the gas phase atomic $\mathrm{D} / \mathrm{H}$ ratio increases with the $\mathrm{CO}$ depletion. Molecules that are formed in the earlier stages of star formation, when the $\mathrm{CO}$ depletion is low, would, therefore, show lower deuteration. Based on our astrochemical model GRAINOBLE (Taquet et al. 2012a, hereafter TCK12a), we theoretically confirmed this hypothesis by successfully reproducing the observed formaldehyde and methanol deuterations (Taquet et al. 2012b, hereafter TCK12b). The difference in deuteration between these two molecules is explained by the earlier formation of formaldehyde compared to methanol, when the $\mathrm{D} / \mathrm{H}$ is lower. We also demonstrated

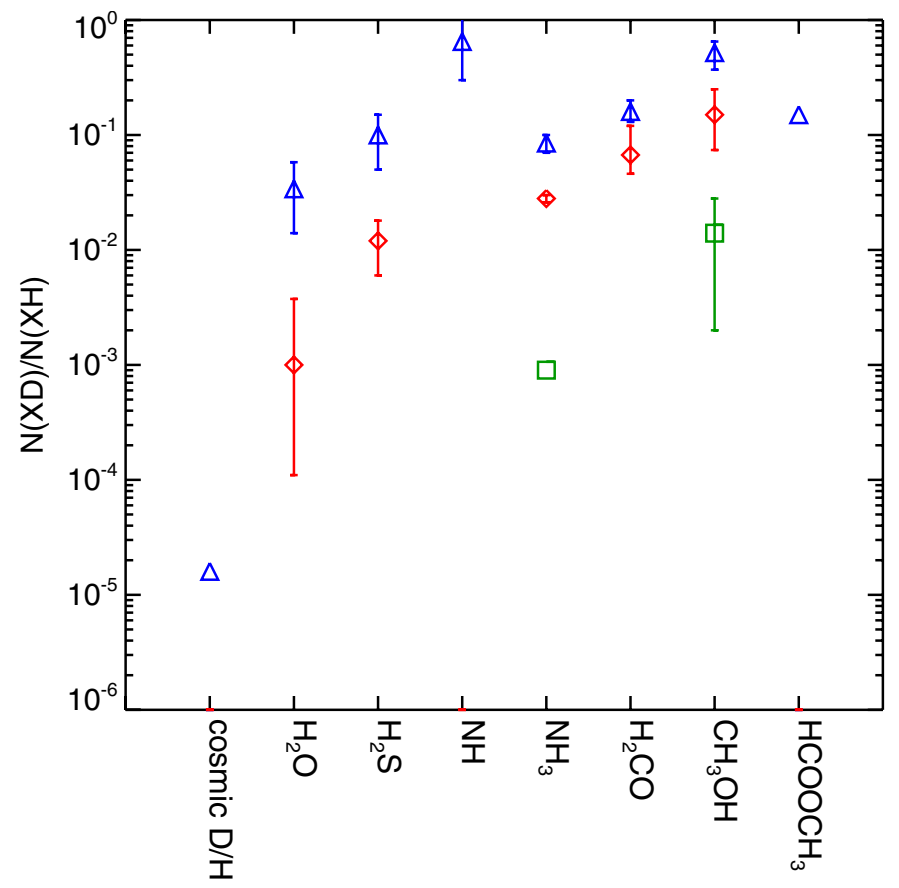

Fig. 1. Deuterium fractionation of several molecules assumed to be partly (or mainly) formed on interstellar grain surfaces and observed around low-mass Class 0 protostars. Blue triangles: simple deuteration; red diamonds: double deuteration; green squares: triple deuteration.

References: Cosmic D/H: Linsky (2003). $\mathrm{H}_{2} \mathrm{O}$ : $\mathrm{HDO} / \mathrm{H}_{2} \mathrm{O}$ by Coutens et al. (2012), $\mathrm{D}_{2} \mathrm{O} / \mathrm{H}_{2} \mathrm{O}$ by Vastel et al. (2010) towards IRAS 16293. $\mathrm{H}_{2} \mathrm{~S}$ : HDS $/ \mathrm{H}_{2} \mathrm{~S}$ by van Dishoeck et al. (1995) towards IRAS 16293 , $\mathrm{D}_{2} \mathrm{~S} / \mathrm{H}_{2} \mathrm{~S}$ by Vastel et al. (2003) towards IRAS $4 \mathrm{~A}$. NH: ND/NH by Bacmann et al. (2010) towards IRAS 16293. $\mathrm{NH}_{3}: \mathrm{NH}_{2} \mathrm{D} / \mathrm{NH}_{3}$ by van Dishoeck et al. (1995) towards IRAS 16293; $\mathrm{NHD}_{2} / \mathrm{NH}_{3}$ by Loinard et al. (2001) towards IRAS 16293 , and $\mathrm{ND}_{3} / \mathrm{NH}_{3}$ by van der Tak et al. (2002) towards IRAS 4A. $\mathrm{H}_{2} \mathrm{CO}: \mathrm{HDCO} / \mathrm{H}_{2} \mathrm{CO}$ by van Dishoeck et al. (1995) towards IRAS 16293 and by Parise et al. (2006) towards seven low-mass protostars; $\mathrm{D}_{2} \mathrm{CO} / \mathrm{H}_{2} \mathrm{CO}$ by Ceccarelli et al. (1998) and Ceccarelli et al. (2001) towards IRAS 16293 and by Parise et al. (2006) and Roberts \& Millar (2007) towards a sample of low-mass protostars. $\mathrm{CH}_{3} \mathrm{OH}: \mathrm{CH}_{2} \mathrm{DOH} / \mathrm{CH}_{3} \mathrm{OH}$ and $\mathrm{CHD}_{2} \mathrm{OH} / \mathrm{CH}_{3} \mathrm{OH}$ by Parise et al. (2006) towards seven low-mass protostars, $\mathrm{CD}_{3} \mathrm{OH} / \mathrm{CH}_{3} \mathrm{OH}$ by Parise et al. (2004) towards IRAS 16293. $\mathrm{HCOOCH}_{3}$ : DCOOCH $3 / \mathrm{HCOOCH}_{3}$ by Demyk et al. (2010) towards IRAS 16293.

the necessity to introduce the abstraction reactions experimentally shown by Nagaoka et al. (2007) and Hidaka et al. (2009). Similarly, Cazaux et al. (2011) showed the possibility that water is formed through reactions involving $\mathrm{H}_{2}$. According to these authors, instead of reflecting the atomic $\mathrm{D} / \mathrm{H}$ ratio, water deuteration should scale with the gas phase $\mathrm{HD} / \mathrm{H}_{2}$ ratio $\left(\sim 10^{-5}\right)$ at low temperatures.

The linear relationship between the observed column density of water ice and the visual extinction, above the threshold of $A_{\mathrm{V}} \sim 3.2 \mathrm{mag}$ found by Whittet et al. (1988), suggests that water ice starts to form significantly at low visual extinctions. Other solid compounds are believed to form along with water ice. The linear relationship between carbon dioxide and water column densities, with a column density ratio $N\left(\mathrm{CO}_{2}\right) / N\left(\mathrm{H}_{2} \mathrm{O}\right)$ of $18 \%$, suggests that these two molecules form in parallel (Whittet et al. 2007). This conclusion is supported by comparisons between observed and laboratory band profiles showing that $\mathrm{CO}_{2}$ is mainly located in a polar water-rich mixture whilst a non-polar component (pure $\mathrm{CO}_{2}$, or $\mathrm{CO}: \mathrm{CO}_{2}$ ) exists in very 
low quantities (Gerakines et al. 1999; Pontoppidan et al. 2008). Other solid organic compounds, such as $\mathrm{CO}$ or $\mathrm{CH}_{3} \mathrm{OH}$, have also been observed in large quantities ( $\sim 30 \%$ for $\mathrm{CO}$, and up to $20 \%$ for methanol) but at higher visual extinctions, above $A_{\mathrm{V}}$ thresholds of 8-9 and 15 mag respectively (Whittet et al. 2007, 2011). Consequently, one needs to include the formation of all other solid molecules in order to correctly study the formation of deuterated water ice.

In this article, we extend our study to the formation and the deuteration of the interstellar water ice formed in molecular clouds and in prestellar cores, using the multilayer GRAINOBLE model. Our goal is to explore the influence of the physical conditions (representative of different typical cloud stages) on ice formation and water deuteration. This is the first time that such a systematic study has been done. Besides that, it includes the crucial influence of the $\mathrm{H}_{2}$ ortho/para ratio on the water deuteration. The astrochemical model is presented in Sect. 2. In Sect. 3, we study the formation of typical grain mantles and show the influence of several physical and chemical parameters on the deuteration of water ice. In each section, we summarise the main ideas with headings and concluding remarks. In Sect. 4, we compare our predictions with previous model predictions and in Sect. 5 with published observations of water, formaldehyde, and methanol.

\section{Modelling}

\subsection{Overview of the GRAINOBLE model}

GRAINOBLE is a gas-grain astrochemical model based on the rate equations approach introduced by Hasegawa et al. (1992). A detailed presentation of GRAINOBLE can be found in TCK12a. Briefly, GRAINOBLE couples gas-phase and grainsurface chemistry. In total, our chemical network consists of 341 (gaseous and solid) species and 3860 (gas phase and grain surface) reactions. The gas phase chemistry is described in detail in Sect. 2.2. The grain surface chemistry processes are the following:

i) The accretion of gas phase species onto the grain surfaces, assumed to be spherical.

ii) The diffusion of adsorbed species via thermal hopping.

iii) The reaction between two particles via the LangmuirHinshelwood mechanism, once they meet in the same site. The reaction rate is the product of the number of times that the two reactants meet each other and the transmission probability $P_{\mathrm{r}}$ of reaction.

iv) The desorption of adsorbed species into the gas phase via several processes:

- thermal desorption;

- cosmic-ray induced heating of grains;

- chemical desorption caused by the energy release of exothermic reactions.

This last process is an upgrade with respect to TCK12a. Following Garrod et al. (2007), we assume a value of 0.012 for the factor $a$ (the ratio of the surface-molecule bond frequency to the frequency at which energy is lost to the grain surface) since it seems to be the most consistent value given by molecular dynamics simulations (Kroes \& Andersson 2005).

In addition, in the present work, we take the effect of the UV photolysis on the ices into account (see Sect. 2.5), because this is important for the formation of $\mathrm{H}_{2} \mathrm{O}$ at low visual extinctions. As suggested by laboratory experiments, cold mantle bulks are mostly inert (see Watanabe et al. 2004; Fuchs et al. 2009; Ioppolo et al. 2010). Therefore, we follow the formation of grain mantles with a multilayer approach in which the outermost only layer is reactive, whilst the mantle bulk remains inert (see TCK12a for more details).

\subsection{Gas-phase chemical network}

We consider the gas-phase chemical network from the KIDA database (Wakelam et al. 2012) for seven elements: H, He, C, $\mathrm{N}, \mathrm{O}, \mathrm{S}$, and Fe, giving a total of 258 gaseous species. Sulphur and iron are introduced to consistently study ion chemistry. Reactions involving atomic $\mathrm{Fe}$ and $\mathrm{S}$ play a significant role in the destruction of $\mathrm{H}^{+}$and $\mathrm{H}_{3}^{+}$whilst $\mathrm{S}^{+}$, and to a lesser extent $\mathrm{Fe}^{+}$, is believed to be one of the most abundant ions before the $\mathrm{CO}$ depletion (see Flower et al. 2005). Relative to the KIDA network, we modify the rate coefficient of the cosmicray dissociation of $\mathrm{H}_{2}$ yielding $\mathrm{H}+\mathrm{H}$. Following Dalgarno et al. (1999), we assume $\gamma=0.5$, where the rate $k_{\text {diss }}\left(\right.$ in s $^{-1}$ ) of cosmic ray dissociation reactions is given by $k_{\mathrm{diss}}=\gamma \zeta$ ( $\zeta$ is the cosmic-ray ionization rate). Before ice formation, bare grains are considered. The recombination efficiency of atomic hydrogen and deuterium are assumed to be unity, following the theoretical works by Cazaux \& Tielens (2004) and Cuppen et al. (2010b), who considered chemisorption interactions. The initial elemental abundances in the gas phase considered in this work are listed in Table 1 and follows the work by Wakelam et al. (2010) and Linsky (2003). To model the water formation at low visual extinctions (see Introduction), we consider the depthdependent self-shielding of $\mathrm{H}_{2}, \mathrm{HD}$, and $\mathrm{CO}$, using the Meudon PDR code (Le Petit et al. 2002, 2006).

Since standard gas-phase models overpredict the $\mathrm{O}_{2}$ abundance with respect to what is observed (see Goldsmith et al. 2011; Liseau et al. 2012) we also ran a grid of models by artificially decreasing the formation rate of $\mathrm{O}_{2}$ by a factor of 10 . We checked that the $\left[\mathrm{O}_{2}\right] /[\mathrm{O}]$ abundance ratio remains indeed ten times lower than the "standard" case throughout the whole calculation.

Deuteration of water and other molecules formed on interstellar grains strongly depend on the gas phase abundances of $\mathrm{H}, \mathrm{D}, \mathrm{H}_{2}, \mathrm{HD}$, and $\mathrm{D}_{2}$. In turn, the abundance of these gaseous species, and more particularly $\mathrm{D}$, depends mainly on the deuteration of $\mathrm{H}_{3}^{+}$which is function of the degree of $\mathrm{CO}$ and $\mathrm{N}_{2}$ freeze-outs onto the grain surfaces (see Roberts \& Millar 2000; Roberts et al. 2003). The deuterium gas phase chemical network is based on that of TCK12b. The major differences with respect to TCK12b are the inclusion of the ortho-to-para (hereinafter opr) $\mathrm{H}_{2}$ ratio and reactions involving $\mathrm{N}_{2}$.

Reactions between $\mathrm{H}_{3}^{+}$isotopologues and $\mathrm{H}_{2}$, reducing the deuterium fractionation, are endothermic. Therefore, these reactions cannot occur in cold-cloud conditions if para- $\mathrm{H}_{2}$ only is considered. However, $\mathrm{H}_{2}$ is also believed to exist in the ortho spin state, higher in energy $(170 \mathrm{~K})$, since $\mathrm{H}_{2}$ probably forms on grains with an ortho-to-para ratio (opr) of 3 . Reactions between ortho- $\mathrm{H}_{2}$ and ortho- $\mathrm{H}_{2} \mathrm{D}^{+}$(ortho- $\mathrm{HD}_{2}^{+}$, ortho- $\mathrm{D}_{3}^{+}$) can reduce the degree of deuteration of $\mathrm{H}_{3}^{+}$significantly at low temperatures. Flower et al. (2006) have shown that the fractionation of $\mathrm{H}_{3}^{+}$, hence, the abundance of atomic $\mathrm{D}$ are strongly reduced when the $\mathrm{H}_{2}$ opr is higher than $10^{-4}$. Consequently, we enlarge the chemical network, relative to TCK12b, by considering the $\mathrm{H}_{3}^{+}-\mathrm{H}_{2}$ system whose new reaction rate coefficients have been computed by Hugo et al. (2009). Ion-neutral reactions between $\mathrm{H}_{3}^{+}$isotopologues (including their different spin states) and $\mathrm{CO}$ or $\mathrm{N}_{2}$, electronic recombinations, and recombinations on electronegative charged grains have also been included following 
Table 1. Initial elemental abundances in the gas phase with respect to hydrogen nuclei (from Linsky 2003; Wakelam et al. 2010).

\begin{tabular}{lc}
\hline \hline Species & Abundance \\
\hline $\mathrm{H}_{2}$ & 0.5 \\
$\mathrm{HD}$ & $1.6 \times 10^{-5}$ \\
$\mathrm{He}$ & 0.09 \\
$\mathrm{C}$ & $1.2 \times 10^{-4}$ \\
$\mathrm{~N}$ & $7.6 \times 10^{-5}$ \\
$\mathrm{O}$ & $2.6 \times 10^{-4}$ \\
$\mathrm{~S}$ & $8.0 \times 10^{-8}$ \\
$\mathrm{Fe}$ & $1.5 \times 10^{-8}$ \\
\hline
\end{tabular}

Roberts \& Millar (2000); Roberts et al. (2003, 2004); Walmsley et al. (2004) and Pagani et al. (2009).

The actual opr of $\mathrm{H}_{2}$ in molecular clouds is still highly uncertain. The initial value of $\mathrm{H}_{2}$ opr formed on grain surfaces is most likely 3 , as recently confirmed by the experiment by Watanabe et al. (2010) conducted on amorphous solid water. Protonexchange reactions in the gas phase would then convert ortho$\mathrm{H}_{2}$ to para- $\mathrm{H}_{2}$, decreasing the opr of $\mathrm{H}_{2}$ towards the Boltzmann value $\left(\sim 3 \times 10^{-7}\right.$ at $10 \mathrm{~K}$, see Flower et al. 2006). Recent experimental studies have also demonstrated that $\mathrm{H}_{2}$ undergoes a nuclear spin conversion from the ortho to the para spin state on amorphous solid water (ASW, Sugimoto \& Fukutani 2011; Chehrouri et al. 2011; Hama et al. 2012). The influence of the $\mathrm{H}_{2}$ opr on absorption lines of formaldehyde has been observed by Troscompt et al. (2009) who deduce that the $\mathrm{H}_{2}$ opr is much lower than 1. Indirect estimates of $\mathrm{H}_{2}$ based on the comparison with chemical models suggest values of about $10^{-3}-10^{-2}$ (Pagani et al. 2009; Dislaire et al. 2012). Given the relative uncertainty in this value and its importance in the molecular deuteration process, in this work we assume the $\mathrm{H}_{2}$ opr as a free parameter constant with time.

\subsection{Chemical network on grain surfaces}

\subsubsection{Formation and deuteration of water ice}

We consider a chemical network based on the work by Tielens \& Hagen (1982) modified following the results of several recent experimental works as described below. The simplest formation pathway towards solid water is the sequential hydrogenation of atomic oxygen:

$\mathrm{O}+\mathrm{H} \rightarrow \mathrm{OH}$

$\mathrm{OH}+\mathrm{H} \rightarrow \mathrm{H}_{2} \mathrm{O}$.

This reaction channel was experimentally measured to occur in cold conditions, probably via barrierless reactions (Hiraoka et al. 1998; Dulieu et al. 2010; Jing et al. 2011).

In addition, water can also be formed from different channels involving $\mathrm{O}_{2}$ or $\mathrm{O}_{3}$ as follows. First, water can be formed from molecular oxygen, either from the gas phase or formed on grains, following the reaction channels:

$\mathrm{O}_{2}+\mathrm{H} \rightarrow \mathrm{HO}_{2}$

$\mathrm{HO}_{2}+\mathrm{H} \rightarrow \mathrm{H}_{2} \mathrm{O}_{2}$

$\mathrm{H}_{2} \mathrm{O}_{2}+\mathrm{H} \rightarrow \mathrm{H}_{2} \mathrm{O}+\mathrm{OH}$.

These pathways have been experimentally demonstrated by Miyauchi et al. (2008); Ioppolo et al. (2010) and Cuppen et al. (2010a) at temperatures of $10 \mathrm{~K}$. Miyauchi et al. (2008) also showed an isotope effect in the formation of water from hydrogen peroxide, implying the possibility of tunnelling through an activation barrier for this reaction.

Second, water can be formed from ozone on interstellar grains (Cuppen \& Herbst 2007; Taquet et al. 2012a) following the reaction

$\mathrm{O}_{3}+\mathrm{H} \rightarrow \mathrm{O}_{2}+\mathrm{OH}$.

Then, $\mathrm{O}_{2}$ and $\mathrm{OH}$ can continue to react to form water via the reactions described above. Mokrane et al. (2009) and Romanzin et al. (2011) experimentally showed the efficiency of this reaction by observing the presence of water $\left(\mathrm{HDO}, \mathrm{D}_{2} \mathrm{O}\right)$ after the irradiation of solid $\mathrm{O}_{3}$ on water ice by $\mathrm{H}$ (D) atoms. We treat this reaction as barrierless.

With their microscopic Monte Carlo model, Cuppen \& Herbst (2007) have concluded that molecular hydrogen plays a key role in the formation of water ice in molecular clouds. When hydrogen is mostly in its molecular form, water is formed at $\sim 70 \%$ by the reaction

$\mathrm{OH}+\mathrm{H}_{2} \rightarrow \mathrm{H}_{2} \mathrm{O}+\mathrm{H}$.

Oba et al. (2012) experimentally showed that HDO formation from $\mathrm{OH}$ is ten times less efficient than the formation of $\mathrm{H}_{2} \mathrm{O}$ from $\mathrm{OH}$, implying an isotope effect and therefore the possibility of tunnelling through an activation barrier in this reaction.

$\mathrm{OH}$ radicals can also recombine if the ice temperature is high enough (40 $\mathrm{K}$ in their experiment) to allow their mobility. Oba et al. (2011) experimentally determined branching ratios for the reactions

$\mathrm{OH}+\mathrm{OH} \rightarrow \mathrm{H}_{2} \mathrm{O}+\mathrm{O}$
$\mathrm{OH}+\mathrm{OH} \rightarrow \mathrm{H}_{2} \mathrm{O}_{2}$

of 0.2 and 0.8 , respectively.

Unlike Cazaux et al. (2010, 2011), we do not include the $\mathrm{O}+\mathrm{H}_{2}$ reaction since it is unlikely to proceed at low temperatures (10-20 K) given its high endothermicity (960 K; Baulch et al. 1992). Oba et al. (2012) confirmed that the co-deposition of cold $\mathrm{O}$ atoms with $\mathrm{H}_{2}$ at $10 \mathrm{~K}$ does not result in the formation of water but only of $\mathrm{O}_{2}$.

We enlarge the water-formation network by including the deuterated counterparts of all reactions mentioned above. Due to the higher mass of deuterated species with respect to their main isotopologue, the reaction rates of barrierless reactions involving deuterated species are decreased. A careful treatment of the transmission probabilities (i.e. probability of tunnelling through the activation barrier) of all reactions possessing a barrier is described in Sect. 2.4.

\subsubsection{Formation and deuteration of other ices}

The accretion of $\mathrm{CO}$ and $\mathrm{O}$ particles onto interstellar grains can lead to the formation of carbon dioxide $\left(\mathrm{CO}_{2}\right)$ mainly via three reaction channels (Ruffle \& Herbst 2001)

$$
\begin{aligned}
& \mathrm{HCO}+\mathrm{O} \rightarrow \mathrm{CO}_{2}+\mathrm{H} \\
& \mathrm{CO}+\mathrm{O} \rightarrow \mathrm{CO}_{2} \\
& \mathrm{CO}+\mathrm{OH} \rightarrow \mathrm{CO}_{2}+\mathrm{H} .
\end{aligned}
$$

Gas phase experiments have shown that reaction (10) is barrierless (e.g. Baulch et al. 2005) although it has never been studied on interstellar ice analogues so far.

Reaction (11) is thought to have a high activation energy (Talbi et al. 2006). Laboratory experiments have shown that solid 
carbon dioxide can be formed from this reaction with low efficiency, at least two orders of magnitude lower than astronomical observations (Roser et al. 2001; Raut \& Baragiola 2011).

Formation of solid carbon dioxide, from $\mathrm{OH}$ radicals and $\mathrm{CO}$ molecules, has been observed even at very low temperatures (10-20 K, Oba et al. 2010; Ioppolo et al. 2011; Noble et al. 2011). However, the exact pathway of the formation of $\mathrm{CO}_{2}$ is still uncertain. Following Oba et al. (2010) who observed a weak band attributed to the HOCO radical and other theoretical works, we propose a reaction pathway for the $\mathrm{CO}_{2}$ formation from $\mathrm{CO}$ and $\mathrm{OH}$, as described in Appendix A.2.

The chemical network presented in TCK12b, with the same relative rates, is used to study the formation and the deuteration of formaldehyde and methanol. We also consider the formation of deuterated methane and ammonia from barrierless addition reactions of solid atomic carbon and nitrogen. A full list of surface reactions considered in this work is presented in Appendix B.

\subsection{Eckart model and reaction probabilities}

In previous gas-grain astrochemical models, the transmission probability of exothermic surface reactions has been approximated by the exponential portion of the quantum mechanical probability for tunnelling through a square potential barrier of width $a$. However, this approach has two main limitations:

i) it does not fit the potential energy profile of reactions correctly;

ii) the width $a$ is unknown, although in most astrochemical models it is arbitrarily fixed to $1 \AA$ (Tielens \& Hagen 1982; Hasegawa et al. 1992). Garrod \& Pauly (2011) reproduced the value of the transmission probability of a few reactions with square potential barriers and deduced a width of about $2 \AA$. However, the deduction of this width is based on poorly constrained activation barriers.

Since square potential barriers do not allow us to accurately estimate the values of the transmission probabilities, we compute the transmission probabilities of all the reactions using the Eckart model (Eckart 1930; Johnston \& Heicklen 1962), which fits an approximate potential energy surface. A full description of the Eckart model, our quantum chemistry calculations and the computations of the transmission probabilities are given in Appendix A.

Briefly, the formation of deuterated water includes two reaction channels which have an activation barrier: reactions (5) and (7). Reaction (5) has been theoretically studied by Koussa et al. (2006) and Ellingson et al. (2007) but only for the main isotopologues. Therefore, quantum chemistry calculations are conducted for all the reactions (5) including deuterated isotopologues (see Appendix A for more details on the calculations). Concerning reaction (7), the transmission probabilities of all the deuterated counterparts have been deduced from the theoretical work by Nguyen et al. (2011).

Figure 2 shows the Eckart and the symmetric square potentials as a function of reaction coordinates for the reaction (5) computed in this work. As suggested previously, it can be seen that the Eckart potential is far to be symmetric. Furthermore, the reaction profile is thinner than the square barrier. Since the quantum tunnelling probability of transmission through this barrier depends on the area of the potential energy profile, the Eckart model gives higher probability of transmission than the square barrier $\left(1.4 \times 10^{-7}\right.$ versus $\left.1.2 \times 10^{-8}\right)$.

Table 2 lists the reactions with an activation barrier, as well as the input parameters needed for computing the transmission

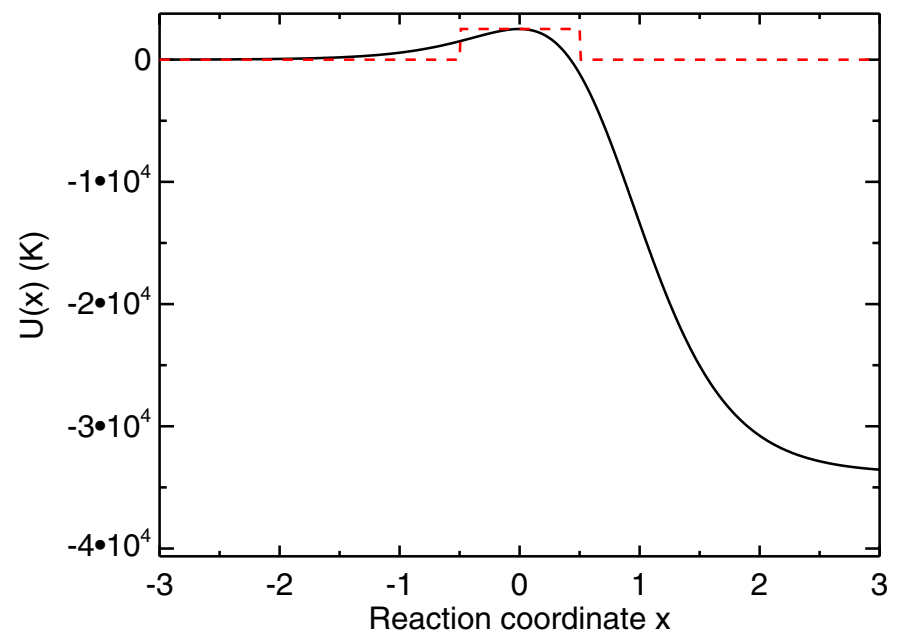

Fig. 2. Potential energy profile as function of the reaction coordinate of the $\mathrm{H}_{2} \mathrm{O}_{2}+\mathrm{H} \rightarrow \mathrm{H}_{2} \mathrm{O}+\mathrm{OH}$ reaction computed from ab-initio calculations (black solid curve) and adopting a symmetric square barrier of a width of $1 \AA$ (red dashed curve). See Appendix A for more details on calculations.

probabilities. It also compares the transmission probability computed with the Eckart model and with a symmetric square potential barrier of the same activation energy and adopting a width of $1 \AA$ (the value commonly used in most gas-grain astrochemical models). The comparisons between the two approaches show that the assumption of a square barrier width of $1 \AA$ tends to underestimate the reaction probabilities for all the reactions by up to seven orders of magnitude.

The transmission probability of the $\mathrm{CO}+\mathrm{H}$ reaction computed with the Eckart model is in good agreement with the range of values we deduced in TCK12a. In this latter work, we varied the transmission probability $P_{\mathrm{r}}$ of this reaction and found that a transmission probability higher than $2 \times 10^{-7}$ was needed to reproduce the solid $\mathrm{CH}_{3} \mathrm{OH} / \mathrm{CO}$ ratio observed towards high-mass protostars.

\subsection{Photodissociation and photodesorption of ices}

In addition to Langmuir-Hinshelwood chemical reactions, we also consider the effect of FUV (6-13.6 eV) photons on ices, following the results of molecular dynamics (MD) simulations carried out by Andersson et al. (2006) and Andersson \& van Dishoeck (2008). They showed that an amorphous ice absorbs UV photons in a 1-2 eV narrow band peaked at $\sim 8.5 \mathrm{eV}$, in good agreement with experimental works by Kobayashi (1983), reaching a maximal absorption probability of $7 \times 10^{-3}$ per monolayer. We convolve the absorption spectrum with the emission spectrum of the interstellar radiation field (ISRF) deduced by Mathis et al. (1983) and with the emission spectrum of $\mathrm{H}_{2}$ excited by the secondary electrons produced by the cosmic-rays ionization of $\mathrm{H}_{2}$ (CRH2RF) computed by Gredel et al. (1987). The absorption probabilities $P_{\text {abs }}$ of each monolayer integrated along the $6-13.6 \mathrm{eV}$ band are therefore equal to $1.51 \times 10^{-3}$ for ISRF and $1.03 \times 10^{-3}$ for CRH2RF. Andersson \& van Dishoeck (2008) also showed that the photofragments, $\mathrm{OH}$ and $\mathrm{H}$, display different trajectories (desorption, trapping, or mobility on the surface) and they computed the yield of each trajectory as function of the monolayer. Subsequent experimental studies by Yabushita et al. (2006, 2009) and Hama et al. (2009, 2010) have confirmed the different photofragment trajectories revealed by 
Table 2. List of reactions having a barrier, with the forward, backward activation barriers, the imaginary frequency of the transition state, the transmission probabilities computed with the Eckart model and the square barrier method (using a barrier width of $1 \AA$ ).

\begin{tabular}{|c|c|c|c|c|c|c|c|c|c|c|c|c|c|}
\hline Reactions & & & & & & & Probability type & $V_{\mathrm{f}}(\mathrm{K})$ & $V_{\mathrm{b}}(\mathrm{K})$ & $v_{\mathrm{S}}$ & $P_{r, \text { Eckart }}$ & $P_{r, \text { square }}$ & Reference \\
\hline $\mathrm{OH}$ & + & $\overline{\mathrm{H}_{2}}$ & $\rightarrow$ & $\mathrm{H}_{2} \mathrm{O}$ & + & $\overline{\mathrm{H}}$ & Eckart & 2935 & 10209 & -1293 & $4.07(-07)$ & $1.49(-13)$ & 1 \\
\hline $\mathrm{OH}$ & + & HD & $\rightarrow$ & $\mathrm{H}_{2} \mathrm{O}$ & + & $\mathrm{D}$ & Eckart & 2855 & 9396 & -1259 & $3.62(-07)$ & 7.91(-16) & 1 \\
\hline $\mathrm{OH}$ & + & HD & $\rightarrow$ & $\mathrm{HDO}$ & + & $\mathrm{H}$ & Eckart & 3051 & 10508 & -970.4 & $1.00(-09)$ & $2.44(-16)$ & 1 \\
\hline $\mathrm{OH}$ & + & $\mathrm{D}_{2}$ & $\rightarrow$ & HDO & + & $\mathrm{D}$ & Eckart & 3026 & 9556 & -955.3 & $8.07(-10)$ & $8.02(-18)$ & 1 \\
\hline OD & + & $\mathrm{H}_{2}$ & $\rightarrow$ & HDO & + & $\mathrm{H}$ & Eckart & 2789 & 10246 & -1293 & $8.74(-07)$ & $2.88(-13)$ & 1 \\
\hline OD & + & HD & $\rightarrow$ & $\mathrm{D}_{2} \mathrm{O}$ & + & $\mathrm{H}$ & Eckart & 2900 & 10871 & -970.1 & $2.81(-09)$ & $5.2(-16)$ & 1 \\
\hline OD & + & $\mathrm{HD}$ & $\rightarrow$ & HDO & + & $\mathrm{D}$ & Eckart & 2703 & 9736 & -1258 & $7.99(-07)$ & $1.76(-15)$ & 1 \\
\hline OD & + & $\mathrm{D}_{2}$ & $\rightarrow$ & $\mathrm{D}_{2} \mathrm{O}$ & + & $\mathrm{D}$ & Eckart & 2870 & 10338 & -955.1 & $2.26(-09)$ & $7.03(-18)$ & 1 \\
\hline$\overline{\mathrm{H}_{2} \mathrm{O}_{2}}$ & + & $\mathrm{H}$ & $\rightarrow$ & $\mathrm{H}_{2} \mathrm{O}$ & + & $\mathrm{OH}$ & Eckart & 2508 & 36358 & -1054 & $1.37(-07)$ & $1.18(-08)$ & 2 \\
\hline $\mathrm{H}_{2} \mathrm{O}_{2}$ & + & $\mathrm{D}$ & $\rightarrow$ & $\mathrm{HDO}$ & + & $\mathrm{OH}$ & Eckart & 2355 & 37118 & -843.7 & $5.54(-09)$ & $8.83(-12)$ & 2 \\
\hline $\mathrm{HDO}_{2}$ & + & $\mathrm{H}$ & $\rightarrow$ & HDO & + & $\mathrm{OH}$ & Eckart & 2523 & 36239 & -1053 & $1.23(-07)$ & $1.17(-08)$ & 2 \\
\hline $\mathrm{HDO}_{2}$ & + & $\mathrm{H}$ & $\rightarrow$ & $\mathrm{H}_{2} \mathrm{O}$ & + & OD & Eckart & 2524 & 36063 & -1053 & $1.22(-07)$ & $1.17(-08)$ & 2 \\
\hline $\mathrm{HDO}_{2}$ & + & D & $\rightarrow$ & $\mathrm{HDO}$ & + & OD & Eckart & 2369 & 36822 & -846.6 & $5.28(-09)$ & $8.66(-12)$ & 2 \\
\hline $\mathrm{HDO}_{2}$ & + & D & $\rightarrow$ & $\mathrm{D}_{2} \mathrm{O}$ & + & $\mathrm{OH}$ & Eckart & 2367 & 37023 & -846.1 & $5.29(-09)$ & $8.66(-12)$ & 2 \\
\hline $\mathrm{D}_{2} \mathrm{O}_{2}$ & + & $\mathrm{H}$ & $\rightarrow$ & $\mathrm{HDO}$ & + & OD & Eckart & 2540 & 35938 & -1052 & $1.08(-07)$ & $1.17(-08)$ & 2 \\
\hline $\mathrm{D}_{2} \mathrm{O}_{2}$ & + & D & $\rightarrow$ & $\mathrm{D}_{2} \mathrm{O}$ & + & OD & Eckart & 2384 & 36721 & -842.9 & $4.28(-09)$ & $8.49(-12)$ & 2 \\
\hline $\mathrm{CO}$ & + & $\mathrm{H}$ & $\rightarrow$ & $\mathrm{HCO}$ & & & Eckart & 1979 & 8910 & -793.6 & $1.92(-07)$ & $1.83(-08)$ & 3 \\
\hline $\mathrm{CO}$ & + & $\mathrm{D}$ & $\rightarrow$ & DCO & & & Experiments & & & & $1.92(-08)$ & $1.83(-09)$ & 4 \\
\hline$\overline{\mathrm{H}_{2} \mathrm{CO}}$ & + & $\mathrm{H}$ & $\rightarrow$ & $\mathrm{CH}_{3} \mathrm{O}$ & & & Experiments & & & & $9.60(-08)$ & $9.15(-09)$ & 4 \\
\hline $\mathrm{H}_{2} \mathrm{CO}$ & + & $\mathrm{D}$ & $\rightarrow$ & $\mathrm{CH}_{2} \mathrm{DO}$ & & & Experiments & & & & $9.60(-09)$ & $9.15(-10)$ & 5 \\
\hline $\mathrm{H}_{2} \mathrm{CO}$ & + & $\mathrm{D}$ & $\rightarrow$ & $\mathrm{HCO}$ & + & HD & Experiments & & & & $9.31(-08)$ & $8.88(-09)$ & 4 \\
\hline $\mathrm{H}_{2} \mathrm{CO}$ & + & $\mathrm{D}$ & $\rightarrow$ & $\mathrm{HDCO}$ & + & $\mathrm{H}$ & Experiments & & & & $9.31(-08)$ & $8.88(-09)$ & 4 \\
\hline HDCO & + & $\mathrm{H}$ & $\rightarrow$ & $\mathrm{CH}_{2} \mathrm{DO}$ & & & Experiments & & & & $1.11(-07)$ & $1.06(-09)$ & 4 \\
\hline HDCO & + & $\mathrm{D}$ & $\rightarrow$ & $\mathrm{CHD}_{2} \mathrm{O}$ & & & Experiments & & & & $1.11(-07)$ & $1.06(-09)$ & 5 \\
\hline $\mathrm{HDCO}$ & + & $\mathrm{H}$ & $\rightarrow$ & $\mathrm{HCO}$ & + & HD & Experiments & & & & $1.54(-07)$ & $1.46(-08)$ & 5 \\
\hline HDCO & + & $\mathrm{D}$ & $\rightarrow$ & DCO & + & HD & Experiments & & & & $9.31(-08)$ & $8.87(-09)$ & 5 \\
\hline HDCO & + & $\mathrm{D}$ & $\rightarrow$ & $\mathrm{D}_{2} \mathrm{CO}$ & + & $\mathrm{H}$ & Experiments & & & & $9.31(-08)$ & $8.87(-09)$ & 5 \\
\hline $\mathrm{D}_{2} \mathrm{CO}$ & + & $\mathrm{H}$ & $\rightarrow$ & $\mathrm{CH}_{2} \mathrm{DO}$ & & & Experiments & & & & $1.27(-07)$ & $1.21(-08)$ & 4 \\
\hline $\mathrm{D}_{2} \mathrm{CO}$ & + & $\mathrm{D}$ & $\rightarrow$ & $\mathrm{CD}_{3} \mathrm{O}$ & & & Experiments & & & & $1.27(-08)$ & $1.21(-09)$ & 5 \\
\hline $\mathrm{D}_{2} \mathrm{CO}$ & + & $\mathrm{H}$ & $\rightarrow$ & DCO & + & HD & Experiments & & & & $7.30(-08)$ & $6.95(-09)$ & 4 \\
\hline $\mathrm{CH}_{3} \mathrm{OH}$ & + & $\mathrm{D}$ & $\rightarrow$ & $\mathrm{CH}_{2} \mathrm{OH}$ & + & HD & Experiments & & & & $2.88(-07)$ & $2.75(-08)$ & 4 \\
\hline $\mathrm{CH}_{2} \mathrm{DOH}$ & + & D & $\rightarrow$ & $\mathrm{CHDOH}$ & + & HD & Experiments & & & & $1.92(-07)$ & $1.83(-08)$ & 4 \\
\hline $\mathrm{CHD}_{2} \mathrm{OH}$ & + & D & $\rightarrow$ & $\mathrm{CD}_{2} \mathrm{OH}$ & + & HD & Experiments & & & & $1.50(-07)$ & $1.43(-08)$ & 4 \\
\hline $\mathrm{CH}_{3} \mathrm{OD}$ & + & D & $\rightarrow$ & $\mathrm{CH}_{2} \mathrm{OD}$ & + & HD & Experiments & & & & $2.88(-07)$ & $2.75(-08)$ & 5 \\
\hline $\mathrm{CH}_{2} \mathrm{DOD}$ & + & $\mathrm{D}$ & $\rightarrow$ & CHDOD & + & $\mathrm{HD}$ & Experiments & & & & $1.92(-07)$ & $1.83(-08)$ & 5 \\
\hline $\mathrm{CHD}_{2} \mathrm{OD}$ & + & D & $\rightarrow$ & $\mathrm{CD}_{2} \mathrm{OD}$ & + & HD & Experiments & & & & $1.50(-07)$ & $1.43(-08)$ & 5 \\
\hline
\end{tabular}

Notes. The probability type refers to the method used for computing the probability. "Experiments" means that the transmission probability is deduced from the $\mathrm{CO}+\mathrm{H}$ reaction by considering the relative rates experimentally measured by Hidaka et al. (2009) (see TCK12b for more details).

References. 1) Nguyen et al. (2011), 2) this work. 3) Peters et al. (2012), 4) Hidaka et al. (2009), 5) Taquet et al. (2012b).

MD simulations. However, our model only considers one chemically reactive layer (the outermost). We deduce the yield of each trajectory by averaging the yields computed by Andersson \& van Dishoeck (2008) on each layer. The list of trajectories as well as their averaged yield $Y$ is given in Table 3 . The rate of each trajectory can be deduced, via the following equation

$R_{\text {photo }, i}\left(\mathrm{~s}^{-1}\right)=Y_{i} \times F_{\mathrm{UV}} \times \sigma\left(a_{\mathrm{d}}\right) \times 5 \times P_{\mathrm{abs}} \times 1 / N_{\mathrm{s}}$

where $F_{\mathrm{UV}}\left(\mathrm{cm}^{-2} \mathrm{~s}^{-1}\right)$ is the photon flux of the $6-13.6 \mathrm{eV} \mathrm{UV}$ band which is absorbed by the amorphous ice, $\sigma\left(a_{\mathrm{d}}\right)\left(\mathrm{cm}^{2}\right)$ is the cross section of interstellar grains $\left(=\pi\left(a_{\mathrm{d}} / 2\right)^{2}\right.$ where $a_{\mathrm{d}}$ is the grain diameter), 5 refers to the absorption of the five outermost layers, and $N_{\mathrm{s}}$ is the number of sites on the grain surface. Owing to the lack of quantitative data on the photodesorption of atoms and on the photodissociation of hydrogenated (and deuterated) molecules other than water on ASW ice, we consider the same absorption probability for all atoms and molecules as for water. For atoms, the desorption probability upon absorption is assumed to be unity, whilst we consider the same outcome probabilities for hydrogenated molecules.
Table 3. List of trajectories after $\mathrm{H}_{2} \mathrm{O}$ photodissociation with their probability (deduced from Andersson \& van Dishoeck 2008, see text).

\begin{tabular}{lc}
\hline \hline Outcome & Probability \\
\hline $\mathrm{H}_{2} \mathrm{O}_{\text {ice }} \rightarrow \mathrm{H}_{\text {gas }}+\mathrm{OH}_{\text {ice }}$ & 0.5 \\
$\mathrm{H}_{2} \mathrm{O}_{\text {ice }} \rightarrow \mathrm{H}_{\text {ice }}+\mathrm{OH}_{\text {ice }}$ & 0.2 \\
$\mathrm{H}_{2} \mathrm{O}_{\text {ice }} \rightarrow \mathrm{H}_{\text {ice }}+\mathrm{OH}_{\text {gas }}$ & $2.2 \times 10^{-3}$ \\
$\mathrm{H}_{2} \mathrm{O}_{\text {ice }} \rightarrow \mathrm{H}_{\text {gas }}+\mathrm{OH}_{\text {gas }}$ & $6.8 \times 10^{-3}$ \\
$\mathrm{H}_{2} \mathrm{O}_{\text {ice }} \rightarrow \mathrm{H}_{2} \mathrm{O}_{\text {gas }}$ & $4.0 \times 10^{-3}$ \\
$\mathrm{H}_{2} \mathrm{O}_{\text {ice }} \rightarrow \mathrm{H}_{2} \mathrm{O}_{\text {ice }}$ & 0.28 \\
\hline
\end{tabular}

We follow the experimental results by Fayolle et al. (2011) for wavelength-dependent $\mathrm{CO}$ photodesorption between 7.5 and $13 \mathrm{eV}$. The convolution of the $\mathrm{CO}$ photodesorption spectrum with the ISRF and CRH2RF fields gives integrated photodesorption yields of $1.2 \times 10^{-2}$ and $9.4 \times 10^{-3}$ photon $^{-1}$ molecule $^{-1}$, respectively. Owing to the lack of data on the wavelengthdependent photodesorption of other molecules, we consider the same photodesorption rates for $\mathrm{O}_{2}$, and $\mathrm{N}_{2}$. 


\subsection{Binding energies}

Comparisons between the observed absorption $3 \mu \mathrm{m}$ band of water and laboratory experiments have shown that grain mantles are mainly composed of high-density amorphous solid water (ASW) (Smith et al. 1989; Jenniskens et al. 1995). Therefore, binding energies of adsorbed species relative to ASW must be considered.

It is now accepted that light particles $\left(\mathrm{H}, \mathrm{D}, \mathrm{H}_{2}, \mathrm{HD}, \mathrm{D}_{2}\right)$ show a distribution of their binding energies relative to ASW, depending on the ice properties, the adsorption conditions, and the coverage of the accreted particles. Perets et al. (2005) have experimentally highlighted the influence of the ice density on the $\mathrm{HD}$ and $\mathrm{D}_{2}$ binding energies. Molecules adsorbed on lowdensity amorphous ices (LDI) desorb following three desorption peaks that are at lower temperatures than the single observed desorption peak of molecules evaporating from a high-density amorphous ice (HDI). By depositing $\mathrm{D}_{2}$ on ASW, Hornekær et al. (2005) showed that $D_{2}$ is more efficiently bound to porous surfaces. Furthermore, binding energies follow broad distributions between 300 and $500 \mathrm{~K}$ and between 400 and $600 \mathrm{~K}$ for non-porous and porous ASW ices, respectively. Amiaud et al. (2006) studied the link between the binding energy distribution of $\mathrm{D}_{2}$ with its coverage on a porous ASW ice and with ice temperature. More particularly, they showed that the $\mathrm{D}_{2}$ binding energy decreases with $\mathrm{H}_{2}$ coverage, from $\sim 700 \mathrm{~K}$ to $\sim 350 \mathrm{~K}$ whilst distributions broaden.

The experimental results have been supported by theoretical MD calculations. For example, Hornekær et al. (2005) and Al-Halabi \& van Dishoeck (2007) found that the binding energy distributions are essentially a consequence of the variation in the number of water molecules surrounding the adsorbed particle. Thus, the binding energy of $\mathrm{H}$ shows a broader distribution peaked at higher values on porous and irregular amorphous water ice than on a structured crystalline ice.

It is believed that deuterated species are more efficiently bound with ices than their main isotopologues because of their higher mass (Tielens 1983). However, the difference in binding energy between $\mathrm{H}, \mathrm{H}_{2}$, and their deuterated counterparts still remains poorly constrained. Experiments by Perets et al. (2005) and Kristensen et al. (2011) have shown that distribution peaks of the $\mathrm{H}_{2}, \mathrm{HD}$, and $\mathrm{D}_{2}$ binding energies are very close (less than $5 \mathrm{meV} \sim 60 \mathrm{~K})$. These differences remain much smaller than the typical full-width-at-half-maximum of binding energy distributions shown by MD simulations $(\sim 195 \mathrm{~K}$ for a amorphous ice Al-Halabi \& van Dishoeck 2007), we, therefore, assume the same binding energy for $\mathrm{H}, \mathrm{D}, \mathrm{H}_{2}, \mathrm{HD}$, and $\mathrm{D}_{2}$. We also verify a posteriori that a small difference of $50 \mathrm{~K}$ between these binding energies has a very limited influence on the deuteration of water.

To take the binding energy distribution of light adsorbed species on ices into account, we assume the binding energy of $\mathrm{H}, \mathrm{D}, \mathrm{H}_{2}, \mathrm{HD}$, and $\mathrm{D}_{2}$ relative to ASW $E_{\mathrm{b}, \mathrm{ASW}}$ as a free parameter between 400 and $600 \mathrm{~K}$. We consider constant binding energies of heavier species, following several experiments. Table 4 lists the binding energies of selected species. We assume that the deuterated species have the same binding energy as their main isotopologue.

$\mathrm{H}_{2}$ is, by about four orders of magnitude, the most abundant molecule in molecular clouds. Most particles that accrete onto the surface are, therefore, $\mathrm{H}_{2}$ molecules. At low temperatures, $\mathrm{H}_{2}$ would become the most abundant icy molecule if binding energies were computed relative to a water ice substrate alone. However, microscopic models by Cuppen \& Herbst (2007) and Cuppen et al. (2009) have shown that the total binding energy
Table 4. List of selected species and binding energies relative to amorphous solid water ice.

\begin{tabular}{lc}
\hline \hline Species & $E_{\mathrm{b}}(\mathrm{K})$ \\
\hline $\mathrm{H}$ & $400-600^{a}$ \\
$\mathrm{H}_{2}$ & $400-600^{a}$ \\
$\mathrm{C}$ & $800^{b}$ \\
$\mathrm{~N}$ & $800^{b}$ \\
$\mathrm{O}$ & $800^{b}$ \\
$\mathrm{CO}$ & $1150^{c}$ \\
$\mathrm{CO}_{2}$ & $2690^{d}$ \\
$\mathrm{O}_{2}$ & $1000^{c}$ \\
$\mathrm{O}_{3}$ & $1800^{e}$ \\
$\mathrm{~N}_{2}$ & $1000^{c}$ \\
$\mathrm{CH}_{4}$ & $1300^{f}$ \\
$\mathrm{NH}_{3}$ & $1300^{f}$ \\
$\mathrm{OH}$ & $2820^{g}$ \\
$\mathrm{H}_{2} \mathrm{O}$ & $5640^{g}$ \\
$\mathrm{H}_{2} \mathrm{O}_{2}$ & $5640^{g}$ \\
$\mathrm{H}_{2} \mathrm{CO}$ & $2050^{f}$ \\
$\mathrm{CH}_{3} \mathrm{OH}$ & $5530^{c}$ \\
\hline
\end{tabular}

References. ${ }^{(a)}$ Hornekær et al. (2005); Al-Halabi \& van Dishoeck (2007); ${ }^{(b)}$ Tielens \& Allamandola (1987); ${ }^{(c)}$ Collings et al. (2004); (d) Sandford \& Allamandola (1990); (e) Cuppen \& Herbst (2007); (f) Garrod \& Herbst (2006); ${ }^{(g)}$ Speedy et al. (1996).

of a adsorbate relative to a substrate is given by the additive energy contribution of the occupied neighbouring sites. Therefore, an $\mathrm{H}_{2} \mathrm{O}: \mathrm{H}_{2}$ mixture must be considered for computing an effective $E_{\mathrm{b}}$. Vidali et al. (1991) gave an estimate of the binding energy of $\mathrm{H}$ on an $\mathrm{H}_{2}$ ice, which is about $45 \mathrm{~K}$ at $10 \mathrm{~K}$. Following Garrod \& Pauly (2011), we compute the effective binding energy of each species $i$ from the fractional coverage of $\mathrm{H}_{2}$ on the surface, $P\left(\mathrm{H}_{2}\right)$

$E_{\mathrm{b}}(i)=\left(1-P\left(\mathrm{H}_{2}\right)\right) E_{\mathrm{b}, \mathrm{wat}}(i)+P\left(\mathrm{H}_{2}\right) E_{\mathrm{b}, \mathrm{H} 2}(i)$

where $E_{\mathrm{b} \text {,wat }}(i)$ is the binding energy of the species $i$ relative to water ice, the values of selected species are listed in Table 4. To deduce $E_{\mathrm{b}, \mathrm{H} 2}$ of species other than $\mathrm{H}$, we apply the same scaling factor as for atomic hydrogen $\left(E_{\mathrm{b}, \mathrm{H} 2}(\mathrm{H}) / E_{\mathrm{b}, \mathrm{ASW}}(\mathrm{H})\right)$. The increase in the $\mathrm{H}_{2}$ abundance in the mantle tends to decrease the effective binding energy $E_{\mathrm{b}}(i)$ of physisorbed species $i$.

\subsection{Physical model}

As described in the introduction, water ice is formed at visual extinctions $A_{\mathrm{V}}$ higher than $3 \mathrm{mag}$ (Whittet et al. 1988, 2001), whilst gas phase $\mathrm{CO}$ is detected above a visual extinction threshold of 2 mag (Frerking et al. 1982) and PDR models show that hydrogen is already mainly molecular at $A_{\mathrm{V}}<1$ mag. Water ice is, therefore, thought to be formed when the gas is already molecular.

We consider a two-step model. For each set of input parameters, we first compute the abundances of gas phase species assuming the element abundances shown in Table 1 and considering the gas phase network described in Sect. 2.2. For this, we assume the steady state values, which are reached after $5 \times 10^{6} \mathrm{yr}$ when the density is $10^{3} \mathrm{~cm}^{-3}$ and after $10^{6} \mathrm{yr}$ at $10^{4} \mathrm{~cm}^{-3}$. This first step is meant to describe the molecular cloud and the initial pre-collapse phase before the formation of the ice bulk. In practice, we assume that the timescale to reach the chemical equilibrium is shorter than the dynamical timescale for the gas to reach the prestellar core conditions. These gas phase abundances are 
then considered as the initial abundances for the gas-grain modelling. In the second step, we allow gas and grain surface chemistry to evolve whilst physical conditions (density, temperature, visual extinction) remain constant.

\subsection{Multi-parameter approach}

Following our previous works (TCK12a, TCK12b), we consider a multi-parameter approach by considering free physical, chemical, and surface input parameters. This approach allows us to study their influence on the formation and the deuteration of key species.

The total density of $\mathrm{H}$ nuclei in molecular clouds, where interstellar ices are thought to be formed, show typical variations from $10^{3} \mathrm{~cm}^{-3}$ at the edge of clouds to $10^{6} \mathrm{~cm}^{-3}$ in denser prestellar cores. Temperatures of the gas and grains also show variations depending on the location inside the cloud. Here, we consider three fixed temperatures, by assuming that the gas and grain surface temperatures are equal, $T_{\mathrm{g}}=T_{\mathrm{d}}$. We study the influence of the visual extinction on the formation and the deuteration of ices by considering $A_{\mathrm{V}}$ as a free parameter. As previously explained in Sect. 2.2, the ortho/para ratio of $\mathrm{H}_{2}$ is considered as constant.

In Sect. 2.6 and in TCK12a and TCK12b, we showed the importance of considering distributions of several grain surface parameters (grain diameter $a_{\mathrm{d}}$, binding energy $E_{\mathrm{b}}$ of light species, diffusion to binding energy ratio $E_{\mathrm{d}} / E_{\mathrm{b}}$ ). We therefore consider them as free parameters, the range of values are listed in Table 5.

We keep fixed the following other parameters:

- distance between two sites $d_{\mathrm{s}}=3.1 \AA$, corresponding to a high-density ASW (Jenniskens et al. 1995);

- cosmic-ray ionization rate $\zeta=3 \times 10^{-17} \mathrm{~s}^{-1}$;

- interstellar radiation field (ISRF) $F_{\text {ISRF }}=1 \times 10^{8}$ photons $\mathrm{cm}^{-2} \mathrm{~s}^{-1}$;

- cosmic ray induced radiation field (CRH2RF) $F_{\mathrm{CRH} 2 \mathrm{RF}}=1 \times$ $10^{4}$ photons $\mathrm{cm}^{-2} \mathrm{~s}^{-1}$

- scaling factor in multiples of the local interstellar field $G_{0}=$ 20 , which represents an average value between low interstellar radiation fields seen in molecular clouds, such as B68 $\left(G_{0}=0.25-1\right.$, Bergin \& Snell 2002), and high ISRFs seen for example, in star-forming molecular clouds in Orion $\left(G_{0}=10^{4}\right.$, Giannini et al. 2000).

In the model grid, some of the parameter values are inconsistent with each other and do not necessarily reflect realistic physical models. We use this model grid in order to systematically study the influence of each parameter on the formation and the deuteration of interstellar ices. For this purpose, we compute the mean values and 1-sigma standard deviations of species abundances and deuterations either in the gas phase or grain surfaces, following the method by Wakelam et al. (2010). Table 5 lists all the free parameters and their ranges of values explored in this work.

\section{Results}

In this section, we present the results in two steps. First, we consider the formation of water and other major ice species, and second, we focus on the deuteration.

Specifically, in Sect. 3.1, we validate our model. For this purpose, i) we compare our predicted gas phase abundances as function of the visual extinction with PDR model predictions; ii) we compare the predicted abundances of gaseous and solid water as function of the visual extinction with published observations;
Table 5. List of the input parameters and the values range explored in this work.

\begin{tabular}{lc}
\hline \hline Input parameters & Values \\
\hline Physical conditions & \\
$n_{\mathrm{H}, \text { ini }}$ & $10^{3}-10^{4}-10^{5}-10^{6} \mathrm{~cm}^{-3}$ \\
$T_{\mathrm{g}}=T_{\mathrm{d}}$ & $10-15-20 \mathrm{~K}$ \\
$A_{\mathrm{V}}$ & $0-1-2-3-5-6-7-8-9-10 \mathrm{mag}$ \\
$\zeta$ & $3 \times 10^{-17} \mathrm{~s}^{-1}$ \\
ISRF & $1 \times 10^{8}$ photons cm ${ }^{-2} \mathrm{~s}^{-1}$ \\
$G_{0}$ & 20 \\
Grain surface parameters & $0.1-\mathbf{0 . 2}-0.3 \mu \mathrm{m}$ \\
$a_{\mathrm{d}}$ & $400-\mathbf{5 0 0}-600 \mathrm{~K}$ \\
$E_{\mathrm{b}}(\mathrm{H})$ & $0.5-\mathbf{0 . 6 5}-0.8$ \\
$E_{\mathrm{d}} / E_{\mathrm{b}}$ & $3.1 \AA$ \\
$d_{\mathrm{s}}$ & \\
Chemical parameters & $3 \times 10^{-6}-3 \times 10^{-4}-3 \times 10^{-2}-3$ \\
$\mathrm{H}_{2} \mathrm{O} / \mathrm{p}$ ratio & \\
\hline
\end{tabular}

Notes. Bold values mark the values adopted in the reference models (see text).

iii) we discuss the multilayer formation of interstellar ices for a set of three reference physical conditions. We emphasize that our approach does not pretend to describe the whole evolution of the cloud. The reference models are meant to quantify the influence of specific physical conditions, which are likely to describe different evolutionary stages, on the ice chemistry.

Second, after validating the model, we focus on the water deuteration. In Sect. 3.2, we emphasize the importance of the CO depletion on the deuterium fractionation of the reference models. Then, in Sect. 3.3, we perform a multiparameter study that allows us to evaluate the influence of each model parameter on the deuterium fractionation of water. Comparing the model predictions with the observations allows us to constrain a range of values for the chemical and physical parameters.

\subsection{Validation of the model}

\subsubsection{Initial gas phase abundances}

As described in Sect. 2.7, we adopt a two-step model. The ice formation phase is followed by considering initial abundances that are computed from steady-state calculations of gas phase chemistry. These initial abundances depend on the density and the temperature, but mainly on the visual extinction $A_{\mathrm{V}}$ (hereafter, $A_{\mathrm{V}}$ means the edge-to-centre visual extinction, half of the observed visual extinction), because photolytic processes play a significant role at low visual extinctions. In fact, the abundance of most gas phase species weakly depends on the density and the temperatures (in the range of values considered in this work) but are essentially a function of the visual extinction. Therefore, we compare the gas-phase abundances of key molecules for ice formation $\left(\mathrm{H}, \mathrm{D}, \mathrm{C}, \mathrm{O}, \mathrm{CO}, \mathrm{O}_{2}\right)$ between our model and the Meudon PDR code (Le Petit et al. 2006) for a total density $n_{\mathrm{H}}=10^{4} \mathrm{~cm}^{-3}$.

Figure 3 shows the depth-dependent chemical abundances of gas phase $\mathrm{H}, \mathrm{D}, \mathrm{O}, \mathrm{C}, \mathrm{O}_{2}$, and $\mathrm{CO}$, for $n_{\mathrm{H}}=10^{4} \mathrm{~cm}^{-3}$ computed by the PDR code in the low visual extinction regime. These abundance profiles are in relative good agreement with the initial abundances computed with our model. The abundances differ by $20 \%$ for most abundant species and within one order of magnitude when species display low abundances $\left(\mathrm{C}\right.$ and $\left.\mathrm{O}_{2}\right)$. This validates the use of the $\mathrm{H}_{2}, \mathrm{HD}$, and $\mathrm{CO}$ self-shieldings in our code. The other differences are due to the different chemical networks 
V. Taquet et al.: Water ice deuteration: a tracer of the chemical history of protostars

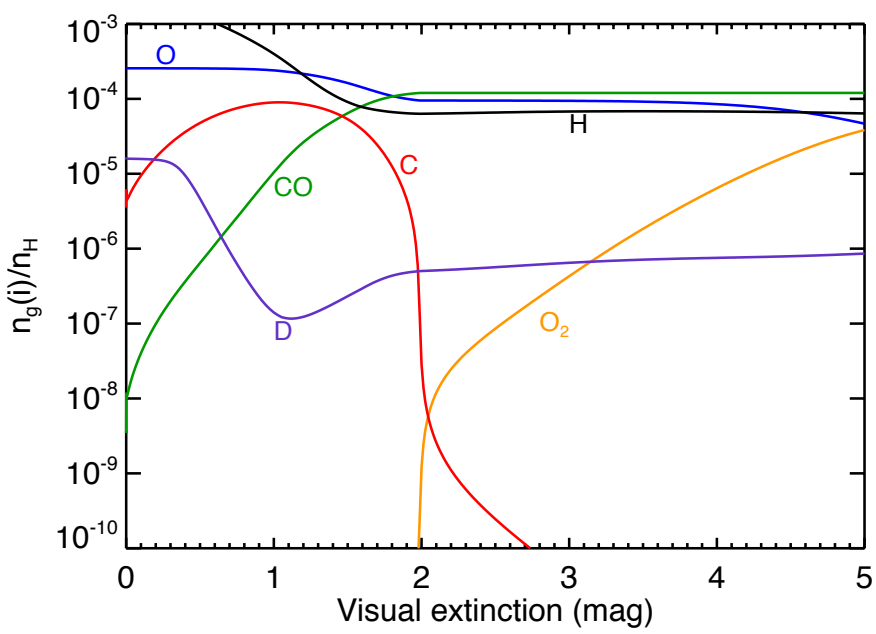

Fig. 3. Initial abundances of $\mathrm{C}, \mathrm{O}, \mathrm{CO}$, and $\mathrm{O}_{2}$ as function of the visual extinction computed with the Meudon PDR code at $n_{\mathrm{H}}=10^{4} \mathrm{~cm}^{-3}$, $G_{0}=20$.

and physical conditions between the two codes (the PDR and ours). The decrease in UV flux with increasing $A_{\mathrm{V}}$ increases the abundance of molecules such as $\mathrm{H}_{2}, \mathrm{HD}$, and $\mathrm{CO}$, and decreases the abundances of $\mathrm{H}, \mathrm{D}$, and then $\mathrm{C}$, and $\mathrm{O}$. D abundance is governed by the high photodissociation of HD at low $A_{\mathrm{V}}$ and by the formation of $\mathrm{H}_{3}^{+}$isotopologues at higher $A_{\mathrm{V}}$, and shows an abundance minimum at $A_{\mathrm{V}}=1 \mathrm{mag}$.

Molecular oxygen is formed at higher visual extinction, and it reaches its maximal abundance at $A_{\mathrm{V}}>5 \mathrm{mag}$. Our chemical network predicts a high $\mathrm{O}_{2} / \mathrm{O}$ abundance ratio $(\sim 1-1.5)$. In fact, the $\mathrm{O}_{2}$ abundance is highly uncertain because it depends on 1) the rate coefficients of some key reactions displaying high uncertainties, and 2) the elemental abundances of carbon and oxygen (see Wakelam et al. 2010; Hincelin et al. 2011). Moreover, observations carried out with the SWASS, Odin, and Herschel space telescopes have revealed that $\mathrm{O}_{2}$ is not abundant in molecular clouds $\left(X\left(\mathrm{O}_{2}\right) \lesssim 10^{-7}\right.$, see Goldsmith et al. 2011; Liseau et al. 2012). Modelling the formation of ices with high $\mathrm{O}_{2}$ abundances is not necessarily realistic, and we, therefore, investigate the influence of the gas phase $\mathrm{O}_{2} / \mathrm{O}$ abundance ratio on the formation of ices in Sect. 3.1.3.

Concluding remarks. As shown in Fig. 3, the chemistry in the gas phase is known to strongly depend on the visual extinction $A_{\mathrm{V}}$. The steady-state gas phase abundances computed with our model are in good agreement with the results of PDR simulations. The use of a two-step model might overestimate the abundance of gaseous $\mathrm{O}_{2}$ affecting ice formation. Therefore, the influence of the $\mathrm{O}_{2} / \mathrm{O}$ ratio on ice formation needs to be investigated.

\subsubsection{Depth-dependent water abundances}

Figure 4 shows the final (at $10^{7} \mathrm{yr}$ ) abundances of water ice and vapour as functions of visual extinction $A_{\mathrm{V}}$ for physical condition representative of molecular clouds (e.g. $n_{\mathrm{H}}=10^{4} \mathrm{~cm}^{-3}$, $\left.T_{\mathrm{g}}=T_{\mathrm{d}}=15 \mathrm{~K}\right)$. The final abundances can be divided into two zones:

i) a photon-dominated layer $\left(A_{\mathrm{V}}<2 \mathrm{mag}\right)$ where gas phase molecules are photodissociated and ices are photodesorbed. At very low visual extinctions, interstellar ices are efficiently photodesorbed and show a low abundance of $\sim 10^{-7}$ relative to $\mathrm{H}$ nuclei corresponding to less than one monolayer. The

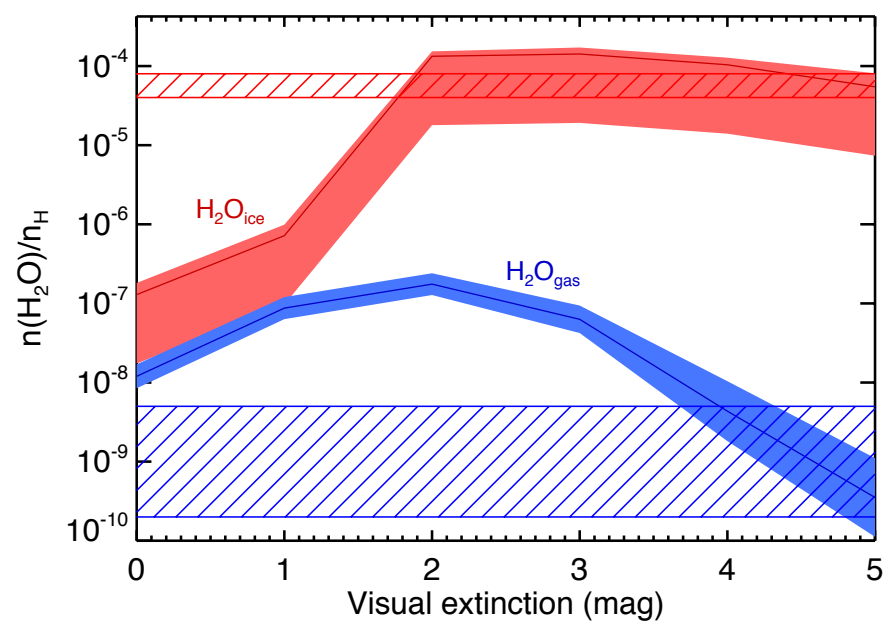

Fig. 4. Final abundances $\left(10^{7} \mathrm{yr}\right)$ of water ice $\left(\mathrm{H}_{2} \mathrm{O}_{\text {ice }}\right.$, red $)$ and water vapour $\left(\mathrm{H}_{2} \mathrm{O}_{\text {gas }}\right.$, blue $)$ as function of the visual extinction $A_{\mathrm{v}}$ for typical molecular cloud conditions $\left(n_{\mathrm{H}}=10^{4} \mathrm{~cm}^{-3}, T=15 \mathrm{~K}\right)$ and including the variation of all other input parameters. Hatched boxes refer to water ice observed by Pontoppidan et al. (2004) towards the Serpens SMM4 regions and water vapour observed by Caselli et al. (2010) towards the L1544 prestellar core (the upper limit represents water abundance in the external part whilst the lower value represents water abundance in the central region).

abundance of water vapour, given by the balance between its formation in gas phase, its photodesorption from interstellar grains, and its photodissociation, reaches steady state values of $10^{-8}-10^{-7}$ (see also Hollenbach et al. 2009).

ii) a darker region $\left(A_{\mathrm{V}}>2 \mathrm{mag}\right)$ where the decrease in UV flux allows the formation of interstellar ices, mainly composed of water. At $A_{\mathrm{V}}=2-4$, water ice is mainly formed from the accretion of $\mathrm{O}$ atoms via the barrierless reaction (2). Most of the oxygen reservoir not trapped in $\mathrm{CO}$ is easily converted into water ice, reaching abundances up to $2 \times 10^{-4}$. The increase in gas-phase $\mathrm{O}_{2}$ initial abundance with $A_{\mathrm{V}}$ slightly decreases the final abundance of water ice. Indeed, water ice is also formed via the accretion of $\mathrm{O}_{2}$ including the formation of hydrogen peroxide. These formation pathways involve reactions possessing significant activation energies and low transmission probabilities (see Table 2). Finally, water formation also depends on the grain surface parameters $E_{\mathrm{d}} / E_{\mathrm{b}}$ and $E_{\mathrm{b}}(\mathrm{H})$. A highly porous case (high $E_{\mathrm{d}} / E_{\mathrm{b}}$ ratio combined with a high binding energy of $\mathrm{H}$ ) strongly decreases the diffusion rate of mobile $\mathrm{H}$ species, decreasing the final abundance of water ice by one order of magnitude $\left(\sim 10^{-5}\right)$.

Gas phase abundance of water is mainly governed by the balance between photodesorption and accretion. The decrease in UV flux with increasing $A_{\mathrm{V}}$ decreases water ice photodesorption and therefore the final abundance of water vapour from $10^{-7}$ at $A_{\mathrm{V}}=2$ to less than $10^{-9}$ at $A_{\mathrm{V}}=5$. We can note that the multilayer nature of grain mantles and the use of a wavelengthdependent absorption of UV photons from ices give similar results to theoretical PDR studies (e.g. Hollenbach et al. 2009).

In spite of the inevitable approximations of our modelling, our predictions are in good agreement with observations of water ice and water vapour, as shown in Fig. 4.

Concluding remarks. For typical conditions representative of a molecular cloud, we are able to reproduce the observed $A_{\mathrm{V}}$ threshold $\left(A_{\mathrm{V}} \sim 1.5 \mathrm{mag}, A_{\mathrm{V}, \mathrm{obs}} \sim 3 \mathrm{mag}\right)$ and the high abundance of water ice $\left(X \sim 10^{-4}\right)$. The low abundance of water 
vapour observed in molecular clouds is reproduced at higher visual extinctions (4-5 mag).

\subsubsection{Reference models}

Very likely, the different ice components and their relative deuteration are the result of a long history where the physical conditions evolve. Therefore, a model aiming at reproducing the whole set of observations should take this evolution into account. However, before embarking on such a complicated modelling, it is worth while and even important to make clear what the characteristics (specifically, solid species and deuteration) are at each evolutionary step. To this end, we consider three "reference" models in the following: i) translucent cloud model, forming $\mathrm{H}_{2} \mathrm{O}$ and $\mathrm{CO}_{2}$ ices; ii) dark cloud model, allowing the formation of $\mathrm{CO}$ ice; iii) dark core model, showing high depletion of $\mathrm{CO}$. The chemical composition of grain mantles for these reference models are shown in Fig. 5 for the two sets of runs with different $\left[\mathrm{O}_{2}\right] /[\mathrm{O}]$ abundance ratios (see Sect. 2.2).

i) Translucent cloud model where ices are mainly composed of $\mathrm{H}_{2} \mathrm{O}$ and $\mathrm{CO}_{2}: n_{\mathrm{H}}=1 \times 10^{4} \mathrm{~cm}^{-3}, T=15 \mathrm{~K}, A_{\mathrm{V}}=2 \mathrm{mag}$ (corresponding to an observed visual extinction of $4 \mathrm{mag}$ ). For the conditions considered in this model, grain mantles are mainly composed of water and carbon dioxide. After $\mathrm{H}_{2}$, the most abundant species that accrete onto grain surfaces are $\mathrm{H}, \mathrm{O}$, and $\mathrm{CO}$. For these conditions, the initial $\mathrm{O}_{2} / \mathrm{O}$ abundance ratio is low $\left(5 \times 10^{-4}\right)$. The decrease in the $\mathrm{O}_{2} / \mathrm{O}$ ratio, therefore, does not affect the ice composition significantly. $\mathrm{O}_{2}$ is involved in the formation of $\mathrm{O}$ and $\mathrm{CO}$ in the gas phase, and its artificial decrease favours $\mathrm{O}$ instead of $\mathrm{CO}$ and increases the formation of water ice by $25 \%$, increasing the ice thickness. The relatively high grain temperature $(15 \mathrm{~K})$ allows accreted particles to diffuse efficiently and form $\mathrm{H}_{2} \mathrm{O}$ and $\mathrm{CO}_{2}$. At this visual extinction, the UV flux irradiating grain surfaces is high, and volatile species, such as atoms or light stable molecules (CO), are efficiently photodesorbed. Water is the main ice component because it is formed from the barrierless reactions (1) and (2) whilst its other reaction routes are negligible (less than $0.1 \%$ ). Carbon dioxide is mainly formed from the hydrogenation of the O...CO complex and not by the direct reaction between $\mathrm{CO}$ and $\mathrm{OH}$. Indeed, the grain temperature is not high enough to allow a high diffusion of $\mathrm{CO}$ and $\mathrm{OH}$, because of their high binding energy. Instead, $\mathrm{O}$ atoms that are less attractively bound can meet $\mathrm{CO}$ atoms to form the O...CO van der Waals complex. In turn, O...CO readily reacts with $\mathrm{H}$ to form a hot $\mathrm{HO}$...CO* complex that forms $\mathrm{CO}_{2}+\mathrm{H}$. The accretion of $\mathrm{CO}$ and $\mathrm{O}$ also allow the formation of formaldehyde and methanol, but only in low abundances, lower than $1 \%$ compared to solid water whilst a low fraction of hydrogen peroxide is predicted for a "normal" $\mathrm{O}_{2} / \mathrm{O}$ ratio. Indeed, they are formed by reactions involving either heavy atoms or significant activation barriers.

The overall abundance of $\mathrm{CO}_{2}$ relative to $\mathrm{H}_{2} \mathrm{O}$ decreases with time, varying from $30 \%$ at the beginning to less than $1 \%$ at the end. The decrease in the $\mathrm{CO}_{2}$ abundance is due to the increase in $\mathrm{H}$ abundance once interstellar ices start to form, because of the high photodesorption.

Although $\mathrm{H}_{2} \mathrm{O}$ and $\mathrm{CO}_{2}$ are formed in high abundances, $\mathrm{O}$ and $\mathrm{CO}$ are not totally depleted on grains. They still show high gas-phase abundances (about $5 \times 10^{-5}$ relative to $\mathrm{H}$ nuclei) at the end of the ice formation. The predicted $\mathrm{CO}_{2}$ abundance relative to water ice is lower than the observations. However, $\mathrm{CO}_{2}$ formation is very sensitive to the grain temperature (governing the diffusion of $\mathrm{O}$ atoms) and to the hydrogen abundance (governing the reaction rate between $\mathrm{O}$ and $\mathrm{H}$ atoms). A higher temperature, higher visual extinction, and/or higher density would, therefore, tend to increase the abundance of $\mathrm{CO}_{2}$ relative to water.

ii) Dark cloud model where CO ice starts to form with water, forming a CO: $\mathrm{H}_{2} \mathrm{O}$ mixture: $n_{\mathrm{H}}=1 \times 10^{4} \mathrm{~cm}^{-3}, T=10 \mathrm{~K}$, $A_{\mathrm{V}}=4 \mathrm{mag}$ (corresponding to an observed visual extinction of $8 \mathrm{mag}), t=10^{6} \mathrm{yr}$. The low temperature limits the diffusion of $\mathrm{O}$ atoms and heavier species whilst the higher visual extinction allows $\mathrm{CO}$ molecules to stay bound on grain surfaces even if they do not react to form $\mathrm{CO}_{2}$. Therefore, the main components of grain mantles are $\mathrm{H}_{2} \mathrm{O}$, mainly formed via the reaction (2), and $\mathrm{CO}$.

$\mathrm{CO}$ shows an overall abundance relative to water of about $80 \%$, which is more than two times higher than observed abundance ratios of ices (10-30\%, Whittet et al. 2007; Öberg et al. 2011). This is due to the high $\mathrm{O}_{2} / \mathrm{O}$ abundance ratio $(\sim 0.5$ throughout the calculation) that allows the formation of $\mathrm{H}_{2} \mathrm{O}_{2}$, via barrierless hydrogenation reactions, instead of water. $\mathrm{H}_{2} \mathrm{O}_{2}$ is easily trapped within grain mantles before reacting because the reaction destroying hydrogen peroxide has a low transmission probability (see Table 2). The decrease in the $\mathrm{O}_{2} / \mathrm{O}$ ratio by one order of magnitude increases the formation of water, giving a $\mathrm{CO} / \mathrm{H}_{2} \mathrm{O}$ abundance ratio of $30 \%$, whilst the $\mathrm{H}_{2} \mathrm{O}_{2}$ abundance decreases by one order of magnitude. Hydrogen peroxide has recently been observed by Bergman et al. (2011) towards the $\rho$ Oph A dark cloud, confirming its formation in ices and its subsequent sublimation via non-photolytic processes. The low grain temperature favours the formation of formaldehyde and methanol via $\mathrm{CO}$ hydrogenation, compared to the formation of $\mathrm{CO}_{2}$. Formaldehyde shows an unexpected abundance higher than $8 \%$ compared to water ice. Again, the formation efficiency of formaldehyde and methanol strongly depends on the grain temperature and on the total density. For example, a higher temperature and/or higher density would favour $\mathrm{CO}_{2}$ formation instead of $\mathrm{H}_{2} \mathrm{CO}$ and $\mathrm{CH}_{3} \mathrm{OH}$.

A visual extinction of 4 mag decreases the final gas-phase abundances of $\mathrm{O}$ and $\mathrm{CO}$, compared to $A_{\mathrm{V}}=2 \mathrm{mag}$. Indeed, gas-phase $\mathrm{CO}$ reaches an abundance of $5 \times 10^{-6}$ whilst $\mathrm{O}$ abundance decreases to $10^{-7}$ at $10^{7} \mathrm{yr}$. The difference in the two abundances is due to the efficient destruction of $\mathrm{O}$ atoms forming solid water, whilst most $\mathrm{CO}$ molecules do not react and are still able to photodesorb.

iii) Dark core model where most of CO is depleted, allowing the formation of pure $\mathrm{CO}$ ice and solid formaldehyde and methanol: $n_{\mathrm{H}}=10^{5} \mathrm{~cm}^{-3}, T=10 \mathrm{~K}, A_{\mathrm{V}}=10 \mathrm{mag}$ (corresponding to an observed $A_{\mathrm{V}}$ of $20 \mathrm{mag}$ ). The low temperature, the high visual extinction, and the high density allow a significant trapping of $\mathrm{CO}$ molecules in the inner part of grain mantles whilst the formation efficiency of $\mathrm{CO}_{2}$ is low owing to the low temperature. $\mathrm{H}_{2} \mathrm{CO}$ and $\mathrm{CH}_{3} \mathrm{OH}$ are formed via hydrogenation reactions that have high activation barriers. They are, therefore, mainly formed in the outer part of the ice when the $\mathrm{CO}$ depletion is high, which allows an efficient hydrogenation (see TCK12a).

At this visual extinction, solid water is less abundant than hydrogen peroxide because the initial gas-phase $\mathrm{O}_{2}$ abundance is higher than the abundance of atomic oxygen $\left(\mathrm{O}_{2} / \mathrm{O}\right.$ between $1 \sim 3$ throughout the calculation). Water formation is mainly formed through the formation of $\mathrm{H}_{2} \mathrm{O}_{2}$, which involves reactions having high activation barriers. Hydrogen 
V. Taquet et al.: Water ice deuteration: a tracer of the chemical history of protostars
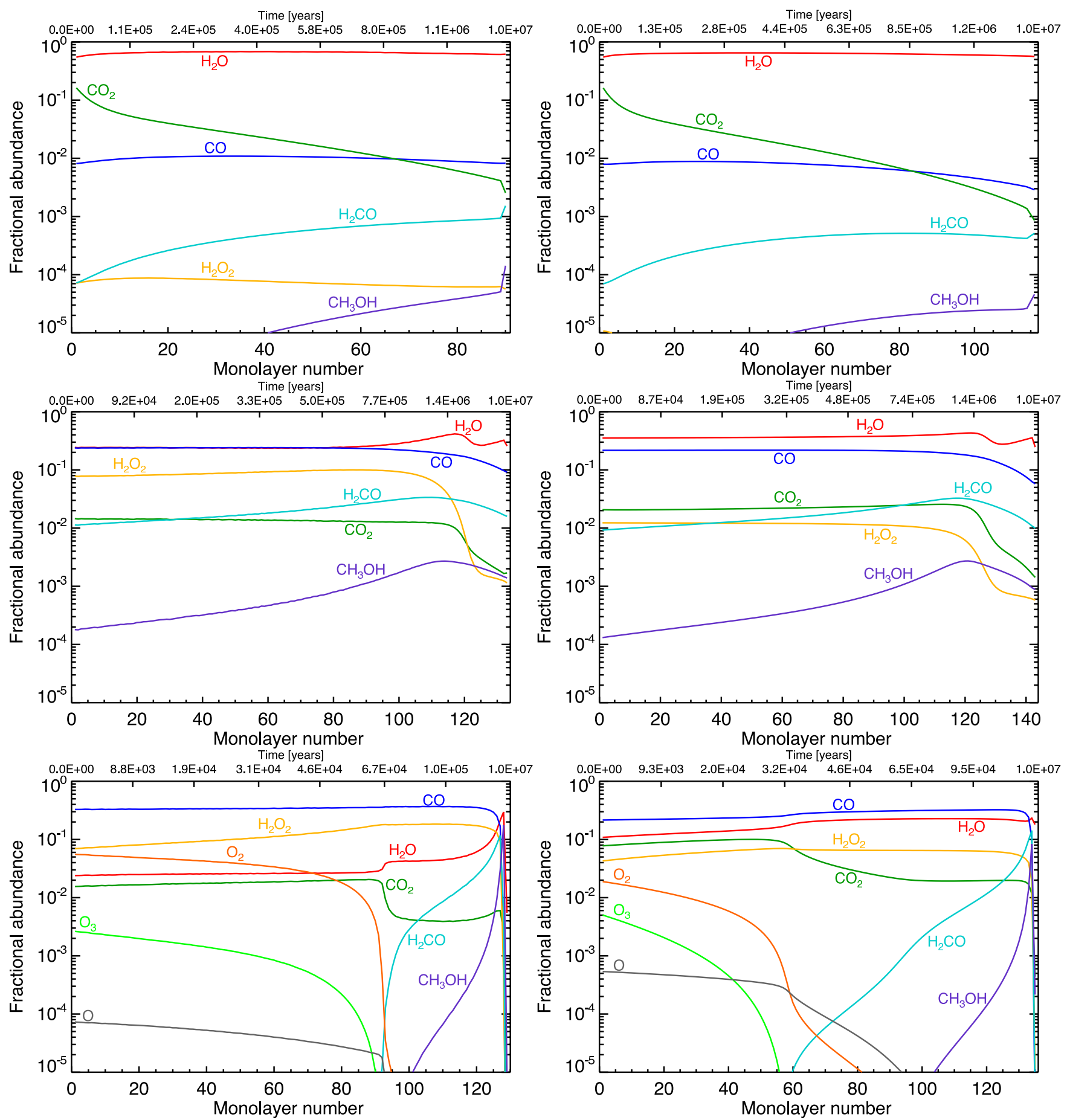

Fig. 5. Fractional ice abundances of main species formed from $\mathrm{O}, \mathrm{O}_{2}$, and $\mathrm{CO}$ for three reference models with "normal" gas phase abundances computed with the chemical network presented in Sect. 2.2 (left), and for a gas phase $\left[\mathrm{O}_{2}\right] /[\mathrm{O}]$ abundance ratio artificially decreased by a factor of 10 (right): top $n_{\mathrm{H}}=1 \times 10^{4} \mathrm{~cm}^{-3}, T=15 \mathrm{~K}, A_{\mathrm{V}}=2 \mathrm{mag}$, middle $n_{\mathrm{H}}=1 \times 10^{4} \mathrm{~cm}^{-3}, T=10 \mathrm{~K}, A_{\mathrm{V}}=4 \mathrm{mag}$, bottom $n_{\mathrm{H}}=1 \times 10^{5} \mathrm{~cm}^{-3}, T=10$ $\mathrm{K}, A_{\mathrm{V}}=10$ mag. Values of other parameters are bold values of Table 5 .

peroxide is most likely trapped in the bulk before forming water owing to the relatively high density. In this case, solid $\mathrm{CO}$ reaches an absolute abundance relative to $\mathrm{H}$ nuclei of $10^{-4}$, water and $\mathrm{CO}_{2}$ show lower abundances $\left(10^{-5}\right.$ and $4 \times 10^{-6}$ ), whilst formaldehyde and methanol show an abundance of $2 \times 10^{-6}$ and $8 \times 10^{-7}$, respectively. Decreasing the $\mathrm{O}_{2} / \mathrm{O}$ abundance ratio to 0.3 strongly increases the formation efficiency of water and $\mathrm{CO}_{2}$ instead of hydrogen peroxide. For this case, water is almost as abundant as CO. Gas-phase abundances of $\mathrm{O}, \mathrm{O}_{2}$, and $\mathrm{CO}$ decrease with time and show high depletions (with final abundances lower than $10^{-10}$ ), whereas $\mathrm{H}$ abundance remains constant.

Concluding remarks. The chemical composition of ices is very sensitive to the physical conditions and to the initial abundances. Most of the observed ice features are reproduced by the reference models: the water-rich ice seen at low visual extinctions is also composed of abundant $\mathrm{CO}_{2}$, whilst the abundances of solid $\mathrm{CO}$ (and $\mathrm{H}_{2} \mathrm{CO}$ and $\mathrm{CH}_{3} \mathrm{OH}$ ) gradually increase with the visual extinction and the density. The increase in the $\mathrm{O}_{2} / \mathrm{O}$ ratio 


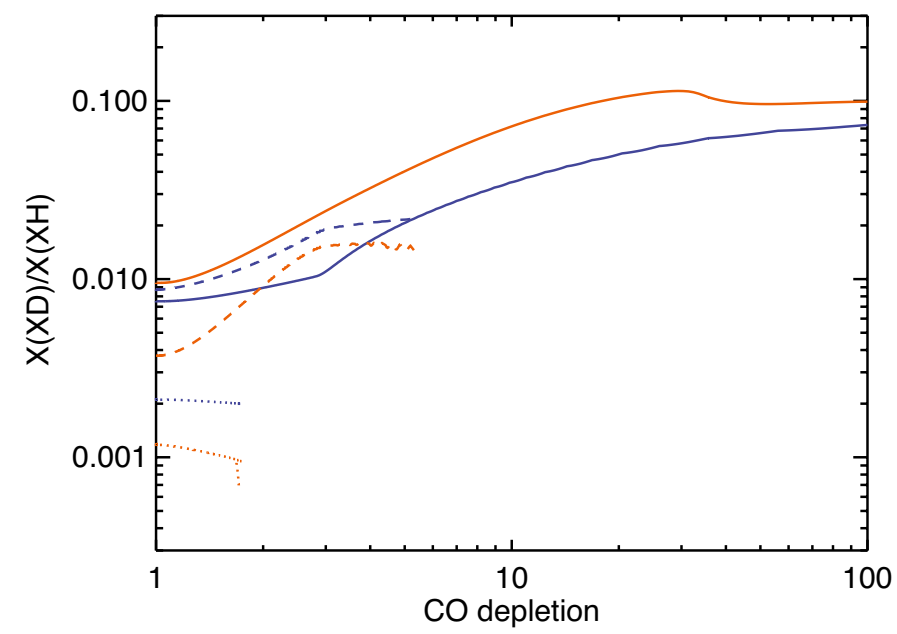

Fig. 6. Solid $\mathrm{HDO} / \mathrm{H}_{2} \mathrm{O}$ (blue) and gaseous $\mathrm{D} / \mathrm{H}$ ratio (orange) as function of the $\mathrm{CO}$ depletion factor $f_{D}(\mathrm{CO})=n_{\mathrm{g}}(\mathrm{CO}) / n_{\mathrm{g}, \text { ini }}(\mathrm{CO})$, which increases with time, for the three reference models: translucent cloud region (dotted), dark cloud region (dashed), dark core region (solid).

decreases the abundance of water because its formation from $\mathrm{O}_{2}$ involves reactions that have significant activation barriers (see Table 2). However, since it is likely that $\mathrm{O}_{2}$ abundance remains low in dark clouds, water formation seems to be efficient in a wide range of physical conditions. Therefore, the study of the water deuteration needs to include the variation of several physical parameters.

\subsection{CO depletion and molecular deuteration}

As discussed in TCK12b, the deuteration of solid species strongly depends on the values of the $\mathrm{CO}$ depletion and the density at the moment of their formation. The initial densities of $\mathrm{H}$ and $\mathrm{D}$ are roughly constant regardless of the total density. Their abundance relative to $\mathrm{H}$ nuclei decreases with increasing $n_{\mathrm{H}}$. At low densities, the increase in $\mathrm{D}$ abundance is limited by the weak deuterium reservoir, whilst the $\mathrm{D} / \mathrm{H}$ ratio is able to strongly increase at higher densities. The gas and grain temperatures, as well as the visual extinction, also affect the evolution of the $\mathrm{D} / \mathrm{H}$ ratio because they influence the desorption rate of $\mathrm{H}$ and D. In summary, since all these quantities vary with time, deuteration is not necessarily constant within grain mantles.

The influence of the CO depletion, the density, the temperatures, and the visual extinction on the water deuteration is shown in Figure 6 for the three "reference" models described in the previous section. At the beginning of the gas-grain calculation, $\mathrm{CO}$ has already reached its maximal abundance $\left(\sim 10^{-4}\right.$, see Fig. 3$)$. With time, the CO molecules freeze-out onto grains, decreasing the gas-phase $\mathrm{CO}$ abundance and increasing the $\mathrm{CO}$ depletion factor. The decrease in the temperatures from 15 to $10 \mathrm{~K}$ and the increase in the visual extinction from 2 to 4 mag increase the gasphase $\mathrm{D} / \mathrm{H}$ ratio from $0.1 \%$ to $0.4 \%$ at $f_{\mathrm{D}}(\mathrm{CO})=1$. The increase in total density from $10^{4}$ to $10^{5} \mathrm{~cm}^{-3}$ increases the final $\mathrm{D} / \mathrm{H}$ ratio from $1 \%$ to $10 \%$. The decrease in the gas-phase $\mathrm{O}_{2} / \mathrm{O}$ abundance ratio does not modify the water deuteration for the two low-density cases. However, it slightly decreases the $\mathrm{HDO} / \mathrm{H}_{2} \mathrm{O}$ ratio for the dense core model by a factor of two because water is more efficiently formed at low CO depletion (see Fig. 5) when the $\mathrm{D} / \mathrm{H}$ ratio is low.

Concluding remarks. As previously noted, water deuteration is largely influenced by the gas $\mathrm{D} / \mathrm{H}$ atomic ratio. Consequently,
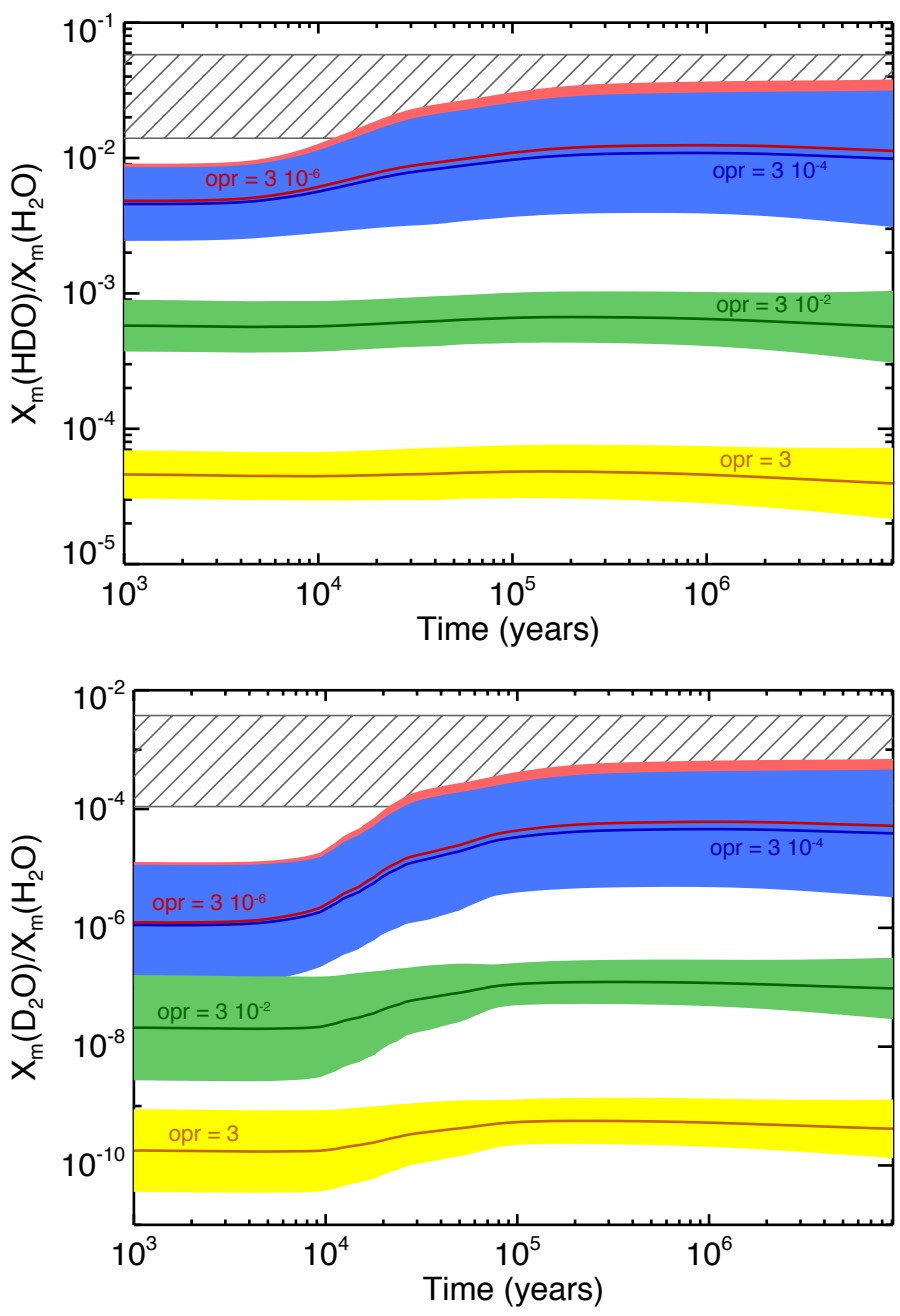

Fig. 7. Deuteration of water ice for four values of $\mathrm{H}_{2}$ ortho/para ratios: $3 \times 10^{-6}$ (red), $3 \times 10^{-4}$ (blue), $3 \times 10^{-2}$ (green), and 3 (yellow) including the variation in all other input parameters, except the visual extinction range that is limited to 2-10 mag. Hatched boxes refer to water deuteration observed by Coutens et al. (2012) towards IRAS 16293.

the largest deuteration is obtained where the $\mathrm{CO}$ depletion and the gas-phase $\mathrm{D} / \mathrm{H}$ ratio are high, namely in the latest and less efficient phases of water formation, represented by the reference model iii). Comparison with the observed deuterium fractionation towards IRAS 16293 shows that a part of water ice should have formed during a dark and/or dense phase. In the following section, we investigate the impact of key parameters on water deuteration.

\subsection{Physical/chemical parameters and water deuteration}

In this section, we study the influence on ice deuteration of several physical and chemical parameters which play a key role. Each figure presented in this section shows the influence of one (or two) parameter(s) at a time. For each value of the studied parameters, mean value and standard deviation of absolute abundances and deuterations induced by the variation in other input parameters are computed. Comparisons between the evolution of the mean deuteration induced by the variation in the studied parameter and the standard deviation caused by other parameters allow us to deduce the importance of that parameter on water ice deuteration. 
V. Taquet et al.: Water ice deuteration: a tracer of the chemical history of protostars
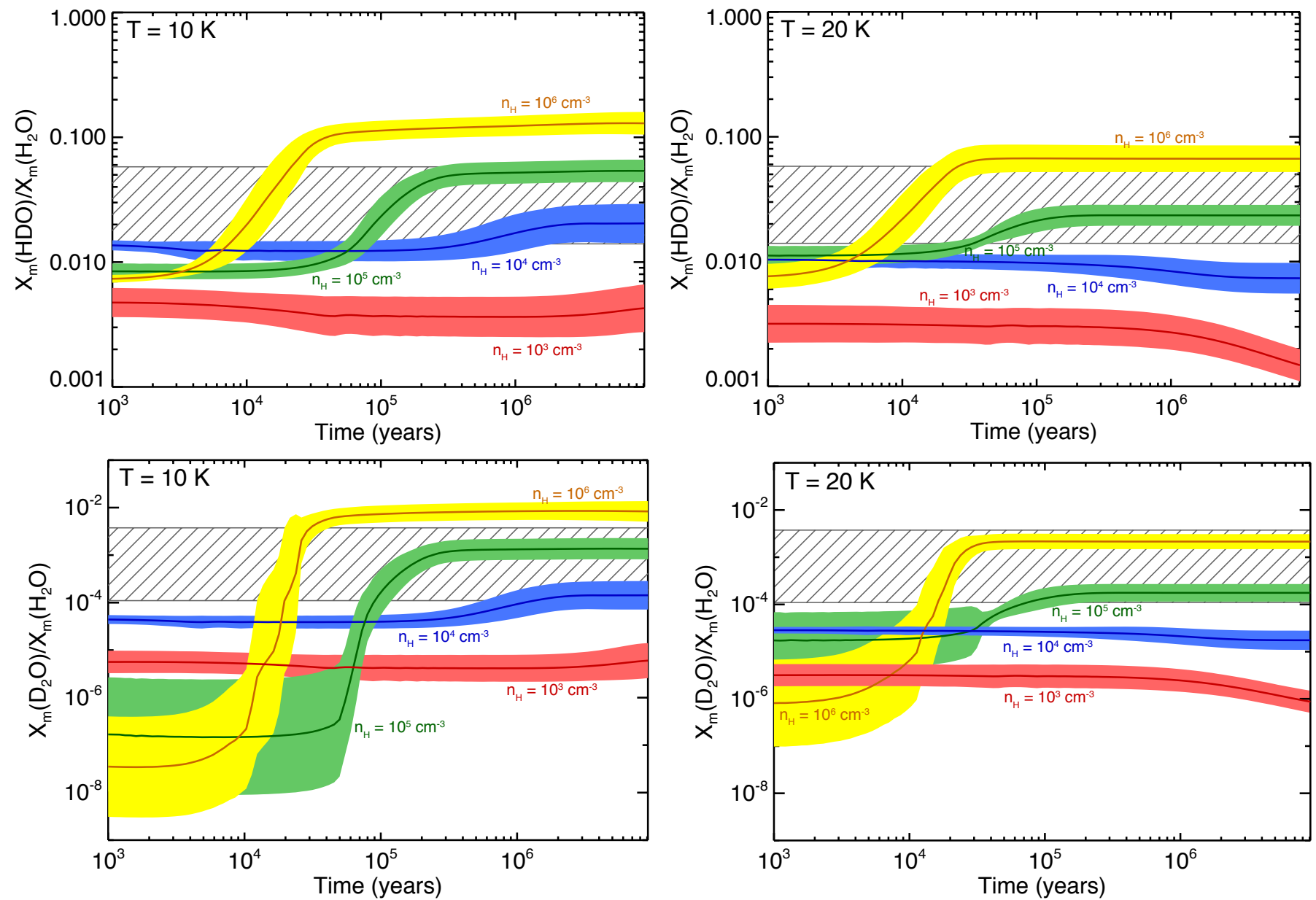

Fig. 8. Deuteration of water ice for four values of total density $10^{3}$ (red), $10^{4}$ (blue), $10^{5}$ (green), $10^{6}$ (yellow) $\mathrm{cm}^{-3}$, and two temperatures: $10 \mathrm{~K}$ (left panels), $20 \mathrm{~K}$ (right panels), using an $\mathrm{H}_{2}$ opr of $3 \times 10^{-6}$, a visual extinction of $10 \mathrm{mag}$, including the variation of all other input parameters. Hatched boxes refer to water deuteration observed by Coutens et al. (2012) towards IRAS 16293.

The predicted deuteration levels of water ice are compared with the observed $\mathrm{HDO} / \mathrm{H}_{2} \mathrm{O}$ and $\mathrm{D}_{2} \mathrm{O} / \mathrm{H}_{2} \mathrm{O}$ ratios towards the hot corino of IRAS 16293 (Coutens et al. 2012) to constrain the input parameters that reproduce the observations best. For this purpose, we assume that the entire bulk of interstellar ices desorbed in the hot corino and the observed deuteration reflects the deuteration in ices (see Introduction).

\subsubsection{Influence of the $\mathrm{H}_{2} \mathrm{o} / \mathrm{p}$ ratio}

As described in Sect. 2.2, the $\mathrm{H}_{2}$ opr influences the deuteration of gas-phase species (including atomic D) (Walmsley et al. 2004; Flower et al. 2006; Pagani et al. 2009), thereby affecting the deuteration of solid water. Figure 7 shows the $\mathrm{HDO} / \mathrm{H}_{2} \mathrm{O}$ and $\mathrm{D}_{2} \mathrm{O} / \mathrm{H}_{2} \mathrm{O}$ ratios in grain mantles as a function of time for four values of $\mathrm{H}_{2}$ opr, including the variation in all other parameters, except the visual extinction range, which is limited to $2-10 \mathrm{mag}$ (where most of water is believed to form, see Fig. 4). As anticipated, the $\mathrm{H}_{2}$ opr governs the deuteration of water ice via its influence on the abundance of $\mathrm{H}_{3}^{+}$isotopologues and atomic $\mathrm{D}$, for values higher than about $3 \times 10^{-4}$. An increase in $\mathrm{H}_{2} \mathrm{opr}$ between $3 \times 10^{-4}$ and 3 decreases the $\mathrm{HDO} / \mathrm{H}_{2} \mathrm{O}$ and $\mathrm{D}_{2} \mathrm{O} / \mathrm{H}_{2}$ ratios by 2.5 orders and 5.5 orders of magnitude, respectively. Furthermore, the ortho/para ratio of $\mathrm{H}_{2}$ is a key, even the most important, parameter for water deuteration. The decrease in the deuteration due to the increase in the $\mathrm{H}_{2}$ opr is much greater than the standard deviation induced by the variation in other parameters.

Comparisons with the observations by Coutens et al. (2012) clearly suggest that a low opr of $\mathrm{H}_{2}$, lower than $3 \times 10^{-4}$, is needed to reproduce the observed deuterium fractionation.

\subsubsection{Influence of the total density}

As discussed in Sect. 3.2, the gas-phase $\mathrm{D} / \mathrm{H}$ ratio is a function of the total density $n_{\mathrm{H}}$ because high densities allow the gas-phase $\mathrm{D} / \mathrm{H}$ ratio to increase with the $\mathrm{CO}$ depletion. Figure 8 shows the deuteration of $\mathrm{HDO}$ and $\mathrm{D}_{2} \mathrm{O}$ for the four considered densities, using an $\mathrm{H}_{2}$ opr of $3 \times 10^{-6}$, two temperatures (10 and $20 \mathrm{~K}$ ), and a high visual extinction (10 mag), including the variation in grain surface parameters.

At low densities $\left(n_{\mathrm{H}} \leq 10^{4} \mathrm{~cm}^{-3}\right)$, water deuteration is constant and low with time because a significant part of HD is already trapped in atomic $\mathrm{D}$, before the $\mathrm{CO}$ depletion. At higher densities $\left(n_{\mathrm{H}}>10^{5} \mathrm{~cm}^{-3}\right)$, the efficient increase in the gaseous $\mathrm{D} / \mathrm{H}$ ratio allows an increase in the deuteration of water up to $10 \%$. In these cases, HDO is mostly located in the outer part of grain mantles, and $\mathrm{D}_{2} \mathrm{O}$ is only located in the outermost layers, whilst $\mathrm{H}_{2} \mathrm{O}$ is present throughout the mantle bulk. For this reason, the $\mathrm{HDO} / \mathrm{H}_{2} \mathrm{O}$ is limited and cannot reach the final gasphase $\mathrm{D} / \mathrm{H}$ ratio (up to $50 \%$ at $n_{\mathrm{H}}=10^{6} \mathrm{~cm}^{-3}$ ). 
In summary, the total density plays a key role in the deuteration of water. Indeed, the variation in the total density $n_{\mathrm{H}}$ between $10^{3}$ and $10^{6} \mathrm{~cm}^{-3}$ influences the $\mathrm{HDO} / \mathrm{H}_{2} \mathrm{O}$ and $\mathrm{D}_{2} / \mathrm{H}_{2} \mathrm{O}$ ratios by 2 and 3.5 orders of magnitude, respectively.

The two observed deuteration ratios can be predicted with total densities $n_{\mathrm{H}}$ between $10^{4}$ and $10^{5} \mathrm{~cm}^{-3}$ at $10 \mathrm{~K}$ and between $10^{5}$ and $10^{6} \mathrm{~cm}^{-3}$ at $20 \mathrm{~K}$, regardless of other grain surface parameters. In the following section, we study the case $n_{\mathrm{H}}=10^{4} \mathrm{~cm}^{-3}$ in more detail to investigate the effect of the temperature and the visual extinction on water deuteration.

\subsubsection{Influence of the temperature and visual extinction}

Temperatures either in the gas-phase and on grain surfaces also influence the deuteration of water. First, an increase in the gasphase temperature enhances the reactivity of endothermic reactions, which can hydrogenate back $\mathrm{H}_{3}^{+}$isotopologues, decreasing the abundance of gaseous D atoms. Second, an increase in the grain temperature severely increases the desorption rate of volatile species, such as atomic H or D. The abundance of gaseous D is limited by the low deuterium reservoir, whereas the abundance of atomic hydrogen can increase up to two orders of magnitude, decreasing the gaseous $\mathrm{D} / \mathrm{H}$ ratio. Water is mainly produced via reactions involving atomic $\mathrm{H}$ and $\mathrm{D}$, increasing gas and dust temperatures, hence, decreasing the water deuteration on grain surfaces.

Figure 9 shows this effect by presenting the final $\mathrm{HDO} / \mathrm{H}_{2} \mathrm{O}$ and $\mathrm{D}_{2} \mathrm{O} / \mathrm{H}_{2} \mathrm{O}$ ratios (at $10^{7} \mathrm{yr}$ ) with the visual extinction, for the three temperatures, considering an $\mathrm{H}_{2}$ opr of $3 \times 10^{-6}$, a total density of $10^{4} \mathrm{~cm}^{-3}$, and including the variation in grain surface parameters. The increase in the (gas and grain) temperatures between 10 and $20 \mathrm{~K}$ slightly decreases the water deuteration approximately by a factor of 3 for $\mathrm{HDO}$ and of 10 for $\mathrm{D}_{2} \mathrm{O}$ at visual extinctions higher than 2 mag. The evolution of deuteration is the same order of magnitude as the standard deviations induced by the variation of other parameters.

The $\mathrm{H}$ and $\mathrm{CO}$ abundances in the gas-phase increase with decreasing $A_{\mathrm{V}}$ because of the increase in the photodesorption rate, decreasing the gaseous $\mathrm{D} / \mathrm{H}$ ratio. Visual extinction, therefore, influences water deuteration at low visual extinctions where water starts to form ( $2 \mathrm{mag}<A_{\mathrm{V}}<5 \mathrm{mag}$ ). As can be seen in Fig. $9, \mathrm{HDO} / \mathrm{H}_{2} \mathrm{O}$ and $\mathrm{D}_{2} \mathrm{O} / \mathrm{H}_{2} \mathrm{O}$ ratios increase by one and two orders of magnitude respectively, between $A_{\mathrm{V}}=2$ and 5 mag.

Comparison with observations shows that observed HDO and $\mathrm{D}_{2} \mathrm{O}$ fractionations are reproduced for a low temperature of $10 \mathrm{~K}$ and for visual extinctions $A_{\mathrm{V}}$ higher than 4 mag $\left(A_{\mathrm{V}, \mathrm{obs}}=\right.$ $8 \mathrm{mag}$ ), implying that deuterated water needs to be formed in dark regions if $n_{\mathrm{H}}=10^{4} \mathrm{~cm}^{-3}$.

\subsubsection{Influence of grain surface parameters}

The grain surface parameters $\left(a_{\mathrm{d}}, E_{\mathrm{d}} / E_{\mathrm{b}}, E_{\mathrm{b}}(\mathrm{H})\right)$ can also influence the formation of interstellar ices. As discussed in TCK12a, the absolute abundance of the main ice constituents formed on grain surfaces (water, formaldehyde, methanol for instance) decrease with $E_{\mathrm{d}} / E_{\mathrm{b}}$ whilst the grain size does not affect the overall abundance (but only the ice thickness). Moreover, it is also seen that the absolute abundance of water slightly decreases with the binding energy of volatile species.

The water deuteration is slightly affected by the variation of the grain surface parameters. As shown in Fig. 9, the standard deviations given by the variation of the grain surface parameters remain lower than the evolution of the water deuteration lead by
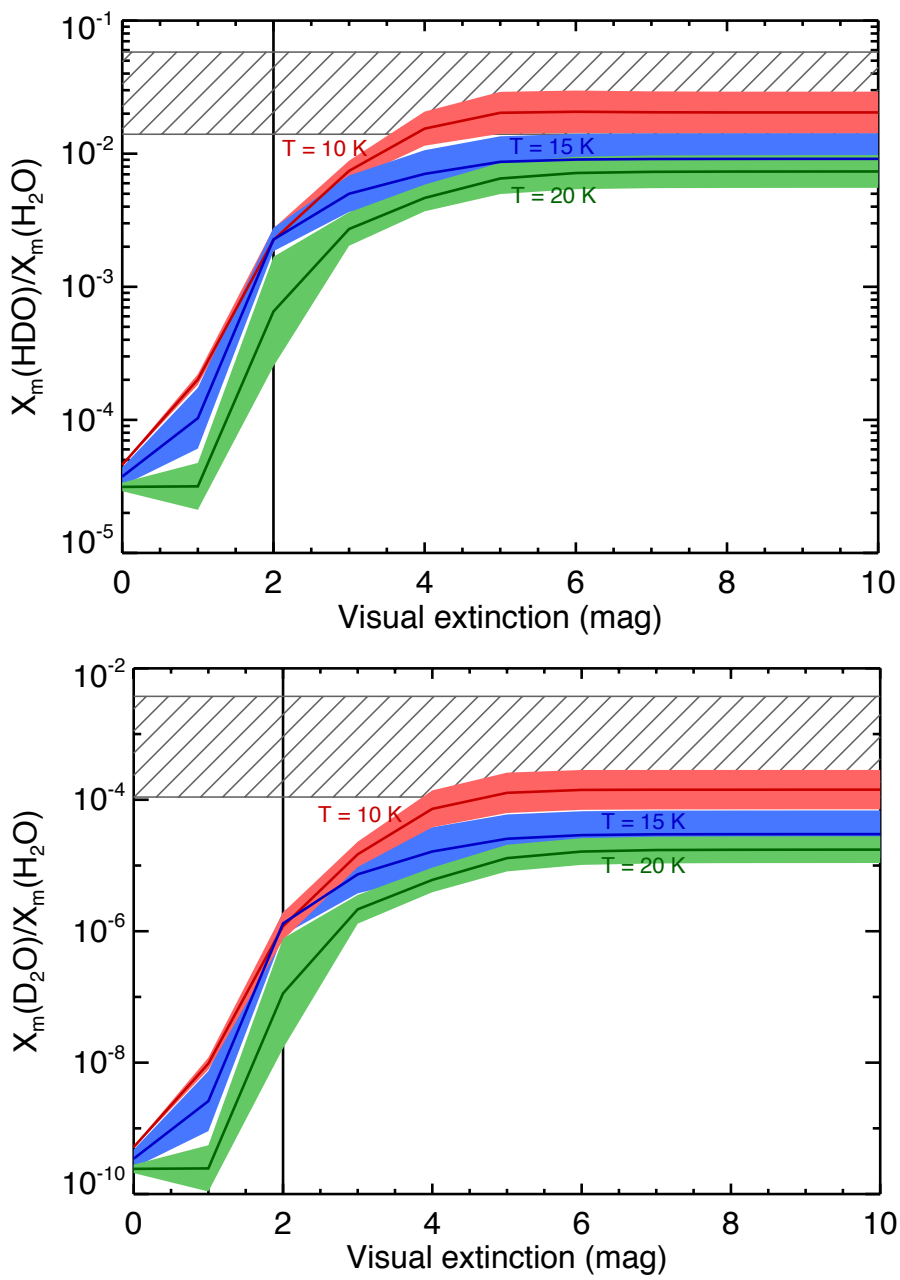

Fig. 9. Final deuteration of water ice (at $t>10^{6} \mathrm{yr}$ : representative of a typical molecular cloud age) as function of visual extinction for three values of temperatures: 10 (red), 15 (blue), 20 (green) $\mathrm{K}$, using a total density of $10^{4} \mathrm{~cm}^{-3}$, an $\mathrm{H}_{2}$ opr of $3 \times 10^{-6}$ and including the variation of all other input parameters. The vertical line refers to the $A_{\mathrm{V}}$ threshold of water ice observed by Whittet et al. (1988). Hatched boxes refer to water deuteration observed by Coutens et al. (2012) towards IRAS 16293.

the variation in the temperature and the visual extinction. The water deuteration slightly decreases with the grain size due to the decrease in the CO depletion efficiency (since the accretion rate is inversely proportional to the grain size), limiting the increase in the deuterium fractionation. On the other hand, the water deuteration slightly increases with $E_{\mathrm{d}} / E_{\mathrm{b}}$ and $E_{\mathrm{b}}(\mathrm{H})$ because the formation efficiency of $\mathrm{H}_{2} \mathrm{O}$ decreases with the diffusion energy of volatile species, increasing the overall fractionation.

\subsubsection{Concluding remarks}

Comparing the average value of the deuterium fractionation with the standard deviation induced by the variation of other parameters allows us to claim that the $\mathrm{H}_{2}$ opr is the most important parameter for water deuteration, followed by the total density. The visual extinction and the temperature also influence water deuteration but more weakly.

The comparison of our model predictions with observations allows us to constrain some parameters involved in the formation 
of deuterated water seen towards IRAS 16293. The observations are reproduced for

- a $\mathrm{H}_{2}$ opr lower than $3 \times 10^{-4}$;

- a density lower than $10^{5} \mathrm{~cm}^{-3}$ if $T=10 \mathrm{~K}$ and $A_{\mathrm{V}}>4 \mathrm{mag}$;

- a higher density $n_{\mathrm{H}}$ between $\sim 5 \times 10^{4} \mathrm{~cm}^{-3}$ and $10^{6} \mathrm{~cm}^{-3}$ if $T=20 \mathrm{~K}$.

\section{Comparisons with previous models}

In this section, we compare our model predictions for the gasphase deuteration and for water ice deuteration with previous models. The atomic $\mathrm{D} / \mathrm{H}$ ratio in the gas-phase depends on the deuteration of $\mathrm{H}_{3}^{+}$. We compare our predictions from steady state models with those by Flower et al. (2006), specifically their Fig. 5. We obtain the same influence of the $\mathrm{H}_{2}$ opr on the deuteration of $\mathrm{H}_{3}^{+}$, with similar fractionation values.

To our knowledge, the only comprehensive theoretical study focused on the deuterated water formation on grain surfaces was performed by Cazaux et al. (2011), even if other works also included the deuteration of water ice in their astrochemical model (i.e. Tielens 1983; Caselli et al. 2002; Stantcheva \& Herbst 2003; Bell et al. 2011). Cazaux et al. (2011) considered a static stage followed by a free-fall collapse phase. However, in their model, the deuterated water is practically formed only during the first static phase. Thus, it is worth comparing their predictions with ours.

The main difference is that they found that the water deuteration is highly temperature-dependent and lower than our predictions at low $(<15 \mathrm{~K})$ temperatures (Fig. 8 of this work versus Fig. 3.c of Cazaux et al. 2011). This is due to a different approach in the reaction probability computation and the use of the endothermic $\mathrm{O}+\mathrm{H}_{2}$ reaction in the chemical network. Cazaux et al. (2011) assumed a competition between the reaction and the diffusion. In their model, if the diffusion timescale of the reactants is larger than the transmission timescale of the reaction, the reaction occurs regardless of its activation barrier. Thus, at low temperatures, the mobility is low and the reaction always occurs. In contrast, in our work, the reaction rate is given by the product of the collision rate of the two reactants and the reaction probability $P_{\mathrm{r}}$ (see Sects. 2.1 and 2.4), giving a much smoother dependence on the temperature. As a consequence, in Cazaux et al. (2011), $\mathrm{H}_{2} \mathrm{O}$ is formed at low temperatures, via reactions involving $\mathrm{H}_{2}$ because of its large abundance, whilst HDO is formed via reactions involving atomic $\mathrm{D}$. The solid $\mathrm{HDO} / \mathrm{H}_{2} \mathrm{O}$ ratio, therefore, scales with the gaseous $[\mathrm{D}] /\left[\mathrm{H}_{2}\right]$ ratio $\left(\sim 10^{-5}\right)$. In our work, water is always mostly formed via the $\mathrm{O}+\mathrm{H}$ and $\mathrm{OH}+\mathrm{H}$ reactions, and the deuteration scales with the $[\mathrm{D}] /[\mathrm{H}]$ ratio (up to $\left.10^{-1}\right)$. At higher temperatures $(>15 \mathrm{~K})$, both models agree since water is formed via the same reactions, namely $\mathrm{O}+\mathrm{H}$ and $\mathrm{OH}+\mathrm{H}$ (and their deuterated counterparts). This comparison shows that the chemical route for the water formation is the major actor in the game, whilst other differences in the two models, including the physical evolution treatment, play a minor role in the water deuteration. However, we should point out that the $\mathrm{O}+\mathrm{H}_{2}$ reaction cannot occur at low temperatures given its endothermicity. Therefore, it is unlikely that water deuteration shows a such strong temperature dependence.

\section{Discussion}

In the previous paragraphs, we have shown that the deuteration of water and other molecules strongly depends on the $\mathrm{H}_{2}$ opr and the total density, but also on the visual extinction and the temperature where solid molecules are formed. The high deuteration of water $\left(\mathrm{HDO} / \mathrm{H}_{2} \mathrm{O}>1 \%\right.$ and $\mathrm{D}_{2} \mathrm{O} / \mathrm{H}_{2} \mathrm{O}>0.01 \%$ ) observed towards the low-mass protostar IRAS 16293 (Coutens et al. 2012) can only be reproduced with an $\mathrm{H}_{2}$ opr that is lower than $3 \times 10^{-4}$, suggesting that most of deuterated water is formed at low $\mathrm{H}_{2}$ opr. The ortho-para ratio reaches its steady-state value on a timescale proportional to the total density, of about $10^{7} \mathrm{yr}$ at $n_{\mathrm{H}}=10^{4} \mathrm{~cm}^{-3}$ (Flower et al. 2006). If the $\mathrm{H}_{2}$ molecule has an initial opr value of 3 upon its formation on grain surfaces, the high deuteration observed around IRAS 16293 would suggest that water observed in this envelope has been formed in an "old" molecular gas, i.e. a gas old enough to show a low $\mathrm{H}_{2}$ opr at the moment of the formation of interstellar ices. However, it is possible that the ortho-to-para conversion also occurs on grain surfaces (Le Bourlot 2000) but with an uncertain rate (see Sugimoto \& Fukutani 2011; Chehrouri et al. 2011; Hama et al. 2012). The decrease in the $\mathrm{H}_{2}$ opr could occur, therefore, faster and the molecular cloud age of $10^{7} \mathrm{yr}$ deduced from the estimate by Flower et al. (2006) should only be used as an upper limit.

The observed deuterium fractionation of water is only reproduced for dark conditions (high visual extinctions and low temperatures if $n_{\mathrm{H}}=10^{4} \mathrm{~cm}^{-3}$ or higher densities). Therefore, although IR observations of ices show that water ice starts to form at low visual extinctions, the deuterated water observed in IRAS 16293 should instead be formed in darker regions. Consequently, $\mathrm{H}_{2} \mathrm{O}$ would be present in the inner part of ice mantles, whilst most of $\mathrm{HDO}$ and $\mathrm{D}_{2} \mathrm{O}$ molecules should be located in the outer layers. A physical evolution, modelling the accumulation of matter from diffuse molecular clouds to dense cores, would allow us to directly confirm this result. The high observed deuteration of water also confirms that water is mostly formed from reactions involving atomic $\mathrm{H}$ and $\mathrm{D}$ and not by molecular hydrogen (see Sect. 4).

Observations of water, formaldehyde, and methanol vapours towards low-mass protostars show that these species have different deuterium fractionations. Figure 10 overplots the observed deuteration towards IRAS 16293 with mean theoretical deuteration values reached at $t=3 \times 10^{5} \mathrm{yr}$ (i.e., the upper limit of the age of prestellar cores, see Bergin \& Tafalla 2007) as function of the density, for two temperatures, a low $\mathrm{H}_{2}$ opr of $3 \times 10^{-6}$ and only considering dark regions $\left(A_{\mathrm{v}}=10 \mathrm{mag}\right)$. The comparison of our predictions with observations shows that

i) our model reproduces the observed $\mathrm{HDO} / \mathrm{H}_{2} \mathrm{O}$ and $\mathrm{D}_{2} \mathrm{O} / \mathrm{H}_{2} \mathrm{O}$ ratios for densities between $1 \times 10^{4}$ and $1 \times$ $10^{5} \mathrm{~cm}^{-3}$ at $10 \mathrm{~K}$ and between and $2 \times 10^{4}$ and $3 \times 10^{5} \mathrm{~cm}^{-3}$ at $20 \mathrm{~K}$. Therefore, water deuteration is reproduced within a wide range of physical conditions representative of molecular clouds but not in the too-translucent cloud phase with too low density, too low visual extinctions and too high temperatures.

ii) the observed $\mathrm{HDCO} / \mathrm{H}_{2} \mathrm{CO}$ can be reproduced at higher densities $\left(3 \times 10^{5}-10^{6} \mathrm{~cm}^{-3}\right)$ and lower temperatures $(\sim 10 \mathrm{~K})$ as seen in the central regions of prestellar cores. The $\mathrm{D}_{2} \mathrm{CO} / \mathrm{H}_{2} \mathrm{CO}$ ratio is reproduced at densities higher than $10^{6} \mathrm{~cm}^{-3}$ (at $\sim 5 \times 10^{6} \mathrm{~cm}^{-3}$, TCK12b).

iii) methanol deuteration $\left(\mathrm{CH}_{2} \mathrm{DOH} / \mathrm{CH}_{3} \mathrm{OH}\right.$ and $\mathrm{CHD}_{2} \mathrm{OH} /$ $\mathrm{CH}_{3} \mathrm{OH}$ ratios) proceeds in the outer parts of grain mantles when the prestellar core condensation reached high densities $\left(>5 \times 10^{5} \mathrm{~cm}^{-3}\right)$ and low temperatures $(10 \mathrm{~K})$.

In summary, the difference in the deuterium fractionation of molecules seen in IRAS 16293 can be explained by different periods of formation. Water is mainly formed first in 


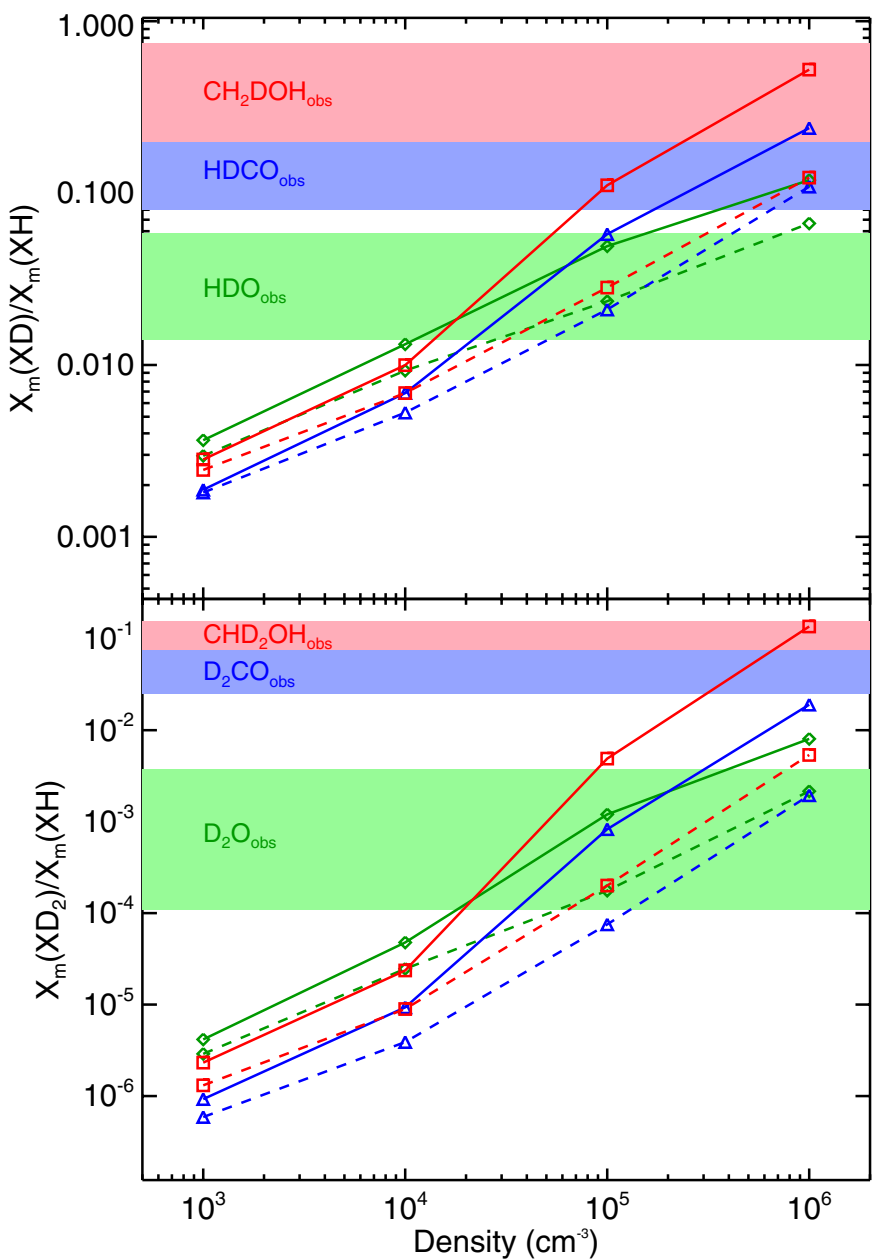

Fig. 10. Averaged deuterations of water (green diamonds), formaldehyde (blue triangles), and methanol (red squares) at $3 \times 10^{5} \mathrm{yr}$ (representative of a typical prestellar core age) as function of the total density (abscissa) and temperature ( $10 \mathrm{~K}$ : solid, $20 \mathrm{~K}$ : dashed) considering an $\mathrm{H}_{2}$ opr of $3 \times 10^{-6}$ and $A_{\mathrm{V}}=10 \mathrm{mag}$ and considering grain surface parameters as free. Solid boxes refer to observations by Coutens et al. (2012) towards IRAS 16293 for water, and by Parise et al. (2006) (and references therein) towards a sample of low-mass protostars for formaldehyde and methanol.

regions showing intermediate densities representative of molecular clouds $\left(n_{\mathrm{H}}=10^{4}-10^{5} \mathrm{~cm}^{-3}\right.$ where the CO depletion is limited. Formaldehyde and methanol are formed subsequently, at higher $\mathrm{CO}$ depletions when the gas is denser and colder. These predictions are in good agreement with infrared observations of interstellar ices presented in the introduction which also show that water ice is formed first, at low visual extinctions, whilst solid methanol forms later on at visual extinctions higher than 15 mag (Whittet et al. 2011).

More generally, the deuterium fractionation of any species, believed to be formed mainly in ices, can be used as a tracer to estimate the physical conditions at the moment of its formation. As shown in Fig. 10, the D/H ratio increases with increasing density and with decreasing temperature. A low deuteration, similar to the deuteration of water, implies that the solid species is mainly formed in regions with relatively low densities, even if its deuterated isotopologues could be formed mainly in a later phase. In contrast, a high deuteration level suggests that the solid species is mostly formed in the dense and cold phase where the deuteration in the gas-phase (and in particular the atomic $\mathrm{D} / \mathrm{H}$ ratio) is high. Extremely high deuteration, such as methanol deuteration, would also imply the existence of abstraction reactions or other processes enhancing the formation of deuterated isotopologues with respect to the main isotopologue (TCK12b). $\mathrm{H} / \mathrm{D}$ exchange reactions between solid methanol and water, for example, can also lead to a selective deuteration of functional groups (Ratajczak et al. 2009).

\section{Conclusions}

We have presented a comprehensive study of the formation and the deuteration of interstellar water ice carried out with our astrochemical model GRAINOBLE. In addition to the multilayer formation of grain mantles presented in a first paper (TCK12a) and the use of abstraction reactions for the deuteration of formaldehyde and methanol (TCK12b), we introduced a better treatment for computing the transmission probabilities of surface reactions based on the Eckart model. We also considered wavelengthdependent UV photodesorption of ices following molecular dynamics (MD) simulations and new experimental works.

The main results of this work are the following:

1) Our model reproduces the abundance of water ice and the visual extinction threshold observed by Whittet et al. (1988), as well as the different ice components seen in different molecular clouds regions.

2) Water deuteration strongly depends on the ortho-to-para ratio of $\mathrm{H}_{2}$ and the total density, but also, even though more weakly, on the gas and grain temperatures and on the visual extinction.

3) The deuteration of water observed towards the low-mass protostar IRAS 16293 can only be reproduced by considering a $\mathrm{H}_{2}$ opr lower than $3 \times 10^{-4}$ and a total density between $8 \times 10^{3}$ and $5 \times 10^{5} \mathrm{~cm}^{-3}$. If a low density $\left(10^{4} \mathrm{~cm}^{-3}\right)$ is considered, a low temperature $(10 \mathrm{~K})$ and a visual extinction higher than 4 mag are necessary. Dark regions (high densities or high visual extinctions) are, therefore, needed to reproduce the observations.

4) Comparison between the observed deuteration of water, formaldehyde, and methanol and our theoretical predictions allowed us to propose the following scenario. Water ice is formed first within relatively wide ranges of physical conditions: $\mathrm{H}_{2} \mathrm{O}$ is allowed to start its formation at low densities and low visual extinctions, but $\mathrm{HDO}$ and $\mathrm{D}_{2} \mathrm{O}$ are instead formed in darker (higher $n_{\mathrm{H}}$ and/or $A_{\mathrm{V}}$ ) regions. Formaldehyde and then methanol are mainly formed subsequently, in the dense and cold prestellar cores.

5) Deuterium fractionation can be used to estimate the values of the density and temperature at the moment of formation of solid species because of its sensitivity to the physical conditions. Low deuteration, similar to water deuteration, implies that the species is formed in a wide range of physical conditions, whilst a high deuteration suggests an efficient formation in the centre of dense and cold prestellar cores.

With this work, we have explored the influence of a wide range of parameters values on the formation of deuterated ices. Now that the influence of the various parameters is clarified, our next step will be a more sophisticated model, based on a realistic physical evolution of a cloud, in a forecoming paper.

Acknowledgements. The authors would like to thank Naoki Watanabe, Yasuhiro Oba, and Tetsuya Hama for fruitful discussions and for their useful comments about the surface reactions occurring in cold conditions, and Guillaume Pineau des Forets for discussions about $\mathrm{H}_{2}$ photodissociation and gas-phase abundance of deuterium. V.T. would like to thank Alexandre Faure for discussions about 
V. Taquet et al.: Water ice deuteration: a tracer of the chemical history of protostars

the $\mathrm{H}_{2}$ ortho/para ratio and a careful reading of the manuscript, and Edith Fayolle for providing useful data on photolysis. This work has been supported by l'Agence Nationale pour la Recherche (ANR), France (project FORCOMS, contracts ANR-08-BLAN-022). A.L.S. and C.C. acknowledge funding from the CNES (Centre National d'Études Spatiales). The computations of the model grid presented in this work were performed at the Service Commun de Calcul Intensif de l'Observatoire de Grenoble (SCCI). Quantum chemical calculations were performed thanks to the HPC resources of CINES under the allocation 2012-088620 made by GENCI (Grand Équipement National de Calcul Intensif). Some kinetic data we used have been downloaded from the online database KIDA (KInetic Database for Astrochemistry, http://kida.obs.u-bordeaux1.fr).

\section{References}

Adamo, C., \& Barone, V. 1999, J. Chem. Phys., 110, 6158

Al-Halabi, A., \& van Dishoeck, E. F. 2007, MNRAS, 382, 1648

Amiaud, L., Fillion, J. H., Baouche, S., et al. 2006, J. Chem. Phys., 124, 094702 Andersson, S., \& van Dishoeck, E. F. 2008, A\&A, 491, 907

Andersson, S., Al-Halabi, A., Kroes, G.-J., \& van Dishoeck, E. F. 2006, J. Chem. Phys., 124, 064715

Andersson, S., Goumans, T., \& Arnaldsson, A. 2011, Chem. Phys. Lett., 513, 31 Andre, P., Ward-Thompson, D., \& Barsony, M. 2000, Protostars and Planets IV, 59

Bacmann, A., Caux, E., Hily-Blant, P., et al. 2010, A\&A, 521, L42

Bates, D. R. 1986, ApJ, 306, L45

Baulch, D. L., Cobos, C. J., Cox, R. A., et al. 1992, J. Phys. Chem. Ref. Data, 21, 411

Baulch, D. L., Bowman, C. T., Cobos, C. J., et al. 2005, J. Phys. Chem. Ref. Data, 34, 757

Bell, T. A., Willacy, K., Phillips, T. G., Allen, M., \& Lis, D. C. 2011, ApJ, 731, 48

Bergin, E. A., \& Snell, R. L. 2002, ApJ, 581, L105

Bergin, E. A., \& Tafalla, M. 2007, ARA\&A, 45, 339

Bergin, E. A., Melnick, G. J., Stauffer, J. R., et al. 2000, ApJ, 539, L129

Bergman, P., Parise, B., Liseau, R., \& Larsson, B. 2011, A\&A, 527, A39

Boogert, A. C. A., Pontoppidan, K. M., Knez, C., et al. 2008, ApJ, 678, 985

Butner, H. M., Charnley, S. B., Ceccarelli, C., et al. 2007, ApJ, 659, L137

Carr, J. S., \& Najita, J. R. 2008, Science, 319, 1504

Caselli, P., Stantcheva, T., Shalabiea, O., Shematovich, V. I., \& Herbst, E. 2002, Planetary and Space Science, 50, 1257

Caselli, P., Keto, E., Pagani, L., et al. 2010, A\&A, 521, L29

Cazaux, S., \& Tielens, A. G. G. M. 2004, ApJ, 604, 222

Cazaux, S., Cobut, V., Marseille, M., Spaans, M., \& Caselli, P. 2010, A\&A, 522, A74

Cazaux, S., Caselli, P., \& Spaans, M. 2011, ApJ, 741, L34

Ceccarelli, C., Hollenbach, D. J., \& Tielens, A. G. G. M. 1996, ApJ, 471, 400

Ceccarelli, C., Castets, A., Loinard, L., Caux, E., \& Tielens, A. G. G. M. 1998, A\&A, 338, L43

Ceccarelli, C., Castets, A., Caux, E., et al. 2000, A\&A, 355, 1129

Ceccarelli, C., Loinard, L., Castets, A., et al. 2001, A\&A, 372, 998

Charnley, S. B., Tielens, A. G. G. M., \& Rodgers, S. D. 1997, ApJ, 482, L203

Chehrouri, M., Fillion, J.-H., Chaabouni, H., et al. 2011, Phys. Chem. Chem. Phys., 13, 2172

Collings, M. P., Anderson, M. A., Chen, R., et al. 2004, MNRAS, 354, 1133

Coutens, A., Vastel, C., Caux, E., \& Ceccarelli, C. 2012, A\&A, 539, A132

Cuppen, H. M., \& Herbst, E. 2007, ApJ, 668, 294

Cuppen, H. M., van Dishoeck, E. F., Herbst, E., \& Tielens, A. G. G. M. 2009, A\&A, 508, 275

Cuppen, H. M., Ioppolo, S., Romanzin, C., \& Linnartz, H. 2010a, Phys. Chem.

Chem. Phys. (Incorporating Faraday Transactions), 12, 12077

Cuppen, H. M., Kristensen, L. E., \& Gavardi, E. 2010b, MNRAS, 406, L11

Dalgarno, A., Yan, M., \& Liu, W. 1999, ApJS, 125, 237

Dartois, E., Thi, W.-F., Geballe, T. R., et al. 2003, A\&A, 399, 1009

Demyk, K., Bottinelli, S., Caux, E., et al. 2010, A\&A, 517, A17

Dislaire, V., Hily-Blant, P., Faure, A., et al. 2012, A\&A, 537, A20

Dulieu, F., Amiaud, L., Congiu, E., et al. 2010, A\&A, 512, A30

Dunning, Jr, T. H. 1989, J. Chem. Phys., 90, 1007

Eckart, C. 1930, Phys. Rev., 35, 1303

Ellingson, B. A., Theis, D. P., Tishchenko, O., Zheng, J., \& Truhlar, D. G. 2007, J. Phys. Chem. A, 111, 13554

Fayolle, E. C., Bertin, M., Romanzin, C., et al. 2011, ApJ, 739, L36

Flower, D. R., Pineau Des Forêts, G., \& Walmsley, C. M. 2005, A\&A, 436, 933

Flower, D. R., Pineau Des Forêts, G., \& Walmsley, C. M. 2006, A\&A, 449, 621

Frerking, M. A., Langer, W. D., \& Wilson, R. W. 1982, ApJ, 262, 590

Frisch, M. J., Trucks, G. W., Schlegel, H. B., et al. 2009, Gaussian 09 Revision A. 02

Fuchs, G. W., Cuppen, H. M., Ioppolo, S., et al. 2009, A\&A, 505, 629

Garrod, R. T., \& Herbst, E. 2006, A\&A, 457, 927
Garrod, R. T., \& Pauly, T. 2011, ApJ, 735, 15

Garrod, R. T., Wakelam, V., \& Herbst, E. 2007, A\&A, 467, 1103

Gerakines, P. A., Whittet, D. C. B., Ehrenfreund, P., et al. 1999, ApJ, 522, 357

Giannini, T., Nisini, B., Lorenzetti, D., et al. 2000, A\&A, 358, 310

Goldsmith, P. F., Liseau, R., Bell, T. A., et al. 2011, ApJ, 737, 96

Goumans, T. P. M., \& Andersson, S. 2010, MNRAS, 406, 2213

Goumans, T. P. M., Uppal, M. A., \& Brown, W. A. 2008, MNRAS, 384, 1158

Gredel, R., Lepp, S., \& Dalgarno, A. 1987, ApJ, 323, L137

Hama, T., Yabushita, A., Yokoyama, M., Kawasaki, M., \& Andersson, S. 2009, J. Chem. Phys., 131, 054508

Hama, T., Yokoyama, M., Yabushita, A., et al. 2010, J. Chem. Phys., 132, 164508

Hama, T., Kuwahata, K., Watanabe, N., et al. 2012, ApJ, 757, 185

Hartogh, P., Lis, D. C., Bockelée-Morvan, D., et al. 2011, Nature, 478, 218

Hasegawa, T. I., \& Herbst, E. M. L. C. 1992, ApJ Suppl. Ser., 82, 167

Hidaka, H., Watanabe, M., Kouchi, A., \& Watanabe, N. 2009, ApJ, 702, 291

Hincelin, U., Wakelam, V., Hersant, F., et al. 2011, A\&A, 530, A61

Hiraoka, K., Miyagoshi, T., Takayama, T., Yamamoto, K., \& Kihara, Y. 1998, ApJ, 498, 710

Hogerheijde, M. R., Bergin, E. A., Brinch, C., et al. 2011, Science, 334, 338

Hollenbach, D., Kaufman, M. J., Bergin, E. A., \& Melnick, G. J. 2009, ApJ, 690, 1497

Hornekær, L., Baurichter, A., Petrunin, V. V., et al. 2005, J. Chem. Phys., 122, 124701

Hugo, E., Asvany, O., \& Schlemmer, S. 2009, J. Chem. Phys., 130, 164302

Ioppolo, S., Cuppen, H. M., Romanzin, C., van Dishoeck, E. F., \& Linnartz, H. 2010, Phys. Chem. Chem. Phys. (Incorporating Faraday Transactions), 12, 12065

Ioppolo, S., van Boheemen, Y., Cuppen, H. M., van Dishoeck, E. F., \& Linnartz, H. 2011, MNRAS, 413, 2281

Jenniskens, P., Blake, D. F., Wilson, M. A., \& Pohorille, A. 1995, ApJ, 455, 389

Jing, D., He, J., Brucato, J., et al. 2011, ApJ, 741, L9

Johnston, H. S., \& Heicklen, J. 1962, J. Phys. Chem., 66, 532

Jørgensen, J. K., \& van Dishoeck, E. F. 2010, ApJ, 725, L172

Kaufman, M. J., \& Neufeld, D. A. 1996, ApJ, 456, 611

Kendall, R. A., Dunning Jr, T. H., \& Harrison, R. J. 1992, J. Chem. Phys., 96, 6796

Klemm, R. B., Payne, W. A., \& Stief, L. J. 1975, in Chemical kinetic data for the upper and lower atmosphere, Proc. Symp., eds. S. W. Benson, D. M. Golden, \& J. R. Barker (New York: John Wiley and Sons)

Klotz, A., Harju, J., Ristorcelli, I., et al. 2008, A\&A, 488, 559

Kobayashi, K. 1983, J. Phys. Chem., 87, 4317

Koussa, H., Bahri, M., Jaidane, N., \& Ben Lakhdar, Z. 2006, J. Mol. Struct. THEOCHEM, 770, 149

Kristensen, L. E., Visser, R., van Dishoeck, E. F., et al. 2010, A\&A, 521, L30

Kristensen, L. E., Amiaud, L., Fillion, J.-H., Dulieu, F., \& Lemaire, J.-L. 2011, A\&A, 527, A44

Kristensen, L. E., van Dishoeck, E. F., Bergin, E. A., et al. 2012, A\&A, 542, A8

Kroes, G. J., \& Andersson, S. 2005, in Astrochemistry: Recent Successes and Current Challenges, eds. D. C. Lis, G. A. Blake, \& E. Herbst, IAU Symp., 231,427

Le Bourlot, J. 2000, A\&A, 360, 656

Le Petit, F., Roueff, E., \& Le Bourlot, J. 2002, A\&A, 390, 369

Le Petit, F., Nehmé, C., Le Bourlot, J., \& Roueff, E. 2006, ApJS, 164, 506

Lebreton, J., Augereau, J.-C., Thi, W.-F., et al. 2012, A\&A, 539, A17

Lefloch, B., Cabrit, S., Codella, C., et al. 2010, A\&A, 518, L113

Li, A., \& Greenberg, J. M. 1998, A\&A, 331, 291

Linsky, J. L. 2003, Space Sci. Rev., 106, 49

Liseau, R., Ceccarelli, C., Larsson, B., et al. 1996, A\&A, 315, L181

Liseau, R., Goldsmith, P. F., Larsson, B., et al. 2012, A\&A, 541, A73

Liu, F.-C., Parise, B., Kristensen, L., et al. 2011, A\&A, 527, A19

Loinard, L., Castets, A., Ceccarelli, C., Caux, E., \& Tielens, A. G. G. M. 2001, ApJ, 552, L163

Mathis, J. S., Mezger, P. G., \& Panagia, N. 1983, A\&A, 128, 212

Miyauchi, N., Hidaka, H., Chigai, T., et al. 2008, Chem. Phys. Lett., 456, 27

Mokrane, H., Chaabouni, H., Accolla, M., et al. 2009, ApJ, 705, L195

Nagaoka, A., Watanabe, N., \& Kouchi, A. 2007, J. Phys. Chem. A, 111, 3016

Nguyen, T. L., Stanton, J. F., \& Barker, J. R. 2011, J. Phys. Chem. A, 115, 5118

Noble, J. A., Dulieu, F., Congiu, E., \& Fraser, H. J. 2011, ApJ, 735, 121

Oba, Y., Watanabe, N., Kouchi, A., Hama, T., \& Pirronello, V. 2010, ApJ, 712, L174

Oba, Y., Watanabe, N., Kouchi, A., Hama, T., \& Pirronello, V. 2011, Phys. Chem. Chem. Phys. (Incorporating Faraday Transactions), 13, 15792

Oba, Y., Watanabe, N., Hama, T., et al. 2012, ApJ, 749, 67

Öberg, K. I., Boogert, A. C. A., Pontoppidan, K. M., et al. 2011, ApJ, 740, 109

Owen, T., \& Bar-Nun, A. 1995, Icarus, 116, 215

Pagani, L., Vastel, C., Hugo, E., et al. 2009, A\&A, 494, 623

Parise, B., Simon, T., Caux, E., et al. 2003, A\&A, 410, 897

Parise, B., Castets, A., Herbst, E., et al. 2004, A\&A, 416, 159 
Parise, B., Caux, E., Castets, A., et al. 2005, A\&A, 431, 547

Parise, B., Ceccarelli, C., Tielens, A. G. G. M., et al. 2006, A\&A, 453, 949 Perdew, J. P., Burke, K., \& Ernzerhof, M. 1996a, Phys. Rev. Lett., 78, 1396 Perdew, J. P., Burke, K., \& Ernzerhof, M. 1996b, Phys. Rev. Lett., 77, 3865 Perets, H. B., Biham, O., Manicó, G., et al. 2005, ApJ, 627, 850

Peters, P. S., Duflot, D., Faure, A., et al. 2011, J. Phys. Chem. A, 115, 8983 Peters, P. S., Duflot, D., Monnerville, M., et al. 2012, J. Phys. Chem. A, submitted

Pontoppidan, K. M., van Dishoeck, E. F., \& Dartois, E. 2004, A\&A, 426, 925 Pontoppidan, K. M., Boogert, A. C. A., Fraser, H. J., et al. 2008, ApJ, 678, 1005 Ratajczak, A., Quirico, E., Faure, A., Schmitt, B., \& Ceccarelli, C. 2009, A\&A, 496, L21

Raut, U., \& Baragiola, R. A. 2011, ApJ, 737, L14

Roberts, H., \& Millar, T. J. 2000, A\&A, 361, 388

Roberts, H., \& Millar, T. J. 2007, A\&A, 471, 849

Roberts, H., Herbst, E., \& Millar, T. J. 2003, ApJ, 591, L41

Roberts, H., Herbst, E., \& Millar, T. J. 2004, A\&A, 424, 905

Romanzin, C., Ioppolo, S., Cuppen, H. M., van Dishoeck, E. F., \& Linnartz, H. 2011, J. Chem. Phys., 134, 084504

Roser, J. E., Vidali, G., Manicò, G., \& Pirronello, V. 2001, ApJ, 555, L61

Ruffle, D., \& Herbst, E. 2001, MNRAS, 324, 1054

Sandford, S. A., \& Allamandola, L. J. 1990, ApJ, 355, 357

Smith, R. G., Sellgren, K., \& Tokunaga, A. T. 1989, ApJ, 344, 413

Speedy, R. J., Debenedetti, P. G., Scott Smith, R., Huang, C., \& Kay, B. D. 1996, J. Chem. Phys., 105, 240

Stantcheva, T., \& Herbst, E. 2003, MNRAS, 340, 983

Sugimoto, T., \& Fukutani, K. 2011, Nat Phys., 7, 307

Talbi, D., Chandler, G., \& Rohl, A. 2006, Chem. Phys., 320, 214

Taquet, V., Ceccarelli, C., \& Kahane, C. 2012a, A\&A, 538, A42

Taquet, V., Ceccarelli, C., \& Kahane, C. 2012b, ApJ, 748, L3

Terada, H., Tokunaga, A. T., Kobayashi, N., et al. 2007, ApJ, 667, 303

Tielens, A. G. G. M. 1983, A\&A, 119, 177
Tielens, A. G. G. M., \& Allamandola, L. J. 1987, in Interstellar Processes, eds. D. J. Hollenbach, \& H. A. Thronson, 397

Tielens, A. G. G. M., \& Hagen, W. 1982, A\&A, 114, 245

Troscompt, N., Faure, A., Maret, S., et al. 2009, A\&A, 506, 1243

van der Tak, F. F. S., Schilke, P., Müller, H. S. P., et al. 2002, A\&A, 388, L53

van Dishoeck, E. F., Blake, G. A., Jansen, D. J., \& Groesbeck, T. D. 1995, ApJ, 447, 760

van Dishoeck, E. F., Kristensen, L. E., Benz, A. O., et al. 2011, PASP, 123, 138

Vastel, C., Phillips, T. G., Ceccarelli, C., \& Pearson, J. 2003, ApJ, 593, L97

Vastel, C., Ceccarelli, C., Caux, E., et al. 2010, A\&A, 521, L31

Vidali, G., Ihm, G., Kim, H., \& Cole, M. 1991, Surf. Sci. Rep., 12, 135

Wakelam, V., Herbst, E., Le Bourlot, J., et al. 2010, A\&A, 517, A21

Wakelam, V., Herbst, E., Loison, J.-C., et al. 2012, ApJS, 199, 21

Walmsley, C. M., Flower, D. R., \& Pineau des Forêts, G. 2004, A\&A, 418, 1035

Wang, H., Eyre, J. A., \& Dorfman, L. M. 1973, J. Chem. Phys., 59, 5199

Watanabe, N., Nagaoka, A., Shiraki, T., \& Kouchi, A. 2004, ApJ, 616, 638

Watanabe, N., Kimura, Y., Kouchi, A., et al. 2010, ApJ, 714, L233

Whittet, D. C. B., \& Duley, W. W. 1991, A\&ARv, 2, 167

Whittet, D. C. B., Bode, M. F., Longmore, A. J., et al. 1988, MNRAS, 233, 321

Whittet, D. C. B., Gerakines, P. A., Hough, J. H., \& Shenoy, S. S. 2001, ApJ, 547,872

Whittet, D. C. B., Shenoy, S. S., Bergin, E. A., et al. 2007, ApJ, 655, 332

Whittet, D. C. B., Cook, A. M., Herbst, E., Chiar, J. E., \& Shenoy, S. S. 2011, ApJ, 742, 28

Woon, D. E. 2002, ApJ, 569, 541

Yabushita, A., Kanda, D., Kawanaka, N., Kawasaki, M., \& Ashfold, M. N. R. 2006, J. Chem. Phys., 125, 133406

Yabushita, A., Hama, T., Yokoyama, M., et al. 2009, ApJ, 699, L80

Yu, H.-G., Muckerman, J. T., \& Sears, T. J. 2001, Chem. Phys. Lett., 349, 547 


\section{Appendix A: Transmission probability computations}

\section{A.1. Eckart model}

We compute the transmission probabilities of all the reactions involved in the water-producing and the methanol-producing networks by using the Eckart model (Eckart 1930; Johnston \& Heicklen 1962). In this approach, an approximate potential energy surface (PES) is fitted as a function of the zero point energies (ZPEs) of the stationary points. The parameters needed for computing the transmission probability are the zero-pointcorrected barrier heights of the forward and reverse reactions $V_{\mathrm{f}}$ and $V_{\mathrm{r}}$, the frequency of the imaginary mode of the transition state $v_{\mathrm{S}}$, and the reduced mass of the reactants $\mu$.

The Eckart potential can be parametrised as

$U_{x}=\frac{A \exp \left(\frac{x-x_{0}}{l}\right)}{1+\exp \left(\frac{x-x_{0}}{l}\right)}+\frac{B \exp \left(\frac{x-x_{0}}{l}\right)}{\left(1+\exp \left(\frac{x-x_{0}}{l}\right)\right)^{2}}$

where

$A=V_{\mathrm{f}}-V_{\mathrm{r}}$

$B=\left(\sqrt{V_{\mathrm{f}}}+\sqrt{V_{\mathrm{r}}}\right)^{2}$

$l=\frac{2 \pi}{\left|\nu_{\mathrm{S}}\right|} \sqrt{\frac{2}{\mu}}\left(\frac{1}{\sqrt{V_{\mathrm{f}}}}+\frac{1}{\sqrt{V_{\mathrm{r}}}}\right)^{-1}$.

Once one has fitted the potential then the transmission probability, $P_{r}$, may be calculated using

$P_{\mathrm{r}}=\frac{\cosh (\alpha+\beta)-\cosh (\alpha-\beta)}{\cosh (\alpha+\beta)+\cosh (\delta)}$

where

$\alpha=\frac{4 \pi}{\left|\nu_{\mathrm{S}}\right|}\left(\frac{1}{\sqrt{V_{\mathrm{f}}}}+\frac{1}{\sqrt{V_{\mathrm{r}}}}\right)^{-1} \sqrt{E}$

$\beta=\frac{8 \pi^{2}}{h\left|\nu_{\mathrm{S}}\right|}\left(\frac{1}{\sqrt{V_{\mathrm{f}}}}+\frac{1}{\sqrt{V_{\mathrm{r}}}}\right)^{-1} \sqrt{E-V_{\mathrm{f}}+V_{\mathrm{r}}}$

$\delta=4 \pi \sqrt{\frac{V_{\mathrm{f}} V_{\mathrm{r}} 2 \pi}{\left(h\left|v_{\mathrm{S}}\right|\right)^{2}}-\frac{1}{16}}$.

When the reactants are considered as excited by their formation involving an exothermic reaction, $E$ refers to the excess energy of this reaction of formation. Otherwise, $E$ is the thermal energy of the particles.

The Eckart model provides a significant improvement over square barriers but it can underpredict (or overpredict) the transmission probabilities of some reactions at low temperatures compared to more exact methods (see Peters et al. 2011). However, given the number of surface reactions considered in this work, exact quantum chemical computations for all reactions are not feasible.

\section{A.2. $\mathrm{CO}_{2}$ formation}

Based on the experimental work by Oba et al. (2010) who studied the formation of $\mathrm{CO}_{2}$ from $\mathrm{CO}$ and $\mathrm{OH}, \mathrm{CO}_{2}$ is thought to be formed via the pathway

$\mathrm{CO}+\mathrm{OH} \rightarrow$ trans-HOCO

trans-HOCO $\rightarrow$ cis-HOCO

cis- $\mathrm{HOCO} \rightarrow \mathrm{CO}_{2}+\mathrm{H}$.
The electronic energy of the intermediate radicals (t-HOCO, $\mathrm{c}-\mathrm{HOCO})$ and the products $\left(\mathrm{CO}_{2}+\mathrm{H}\right)$ are lower than for the reactants $(\mathrm{CO}+\mathrm{OH})$. Reactions involving $\mathrm{t}-\mathrm{HOCO}$ and $\mathrm{c}-\mathrm{HOCO}$ possess high activation barriers and/or are endothermic (Yu et al. 2001). Therefore, $t-H O C O$ radicals continue to react only if their excess energy released by the chemical energy of reaction (A.9) is sufficient to overcome the high activation barriers of reactions (A.10) and (A.11). As in Goumans et al. (2008), HOCO radicals can also react with $\mathrm{H}$ atoms via barrierless reactions to form three pairs of products: $\mathrm{H}_{2}+\mathrm{CO}_{2}, \mathrm{H}_{2} \mathrm{O}+\mathrm{CO}$, or $\mathrm{HCOOH}$. The lack of more quantitative data leads us to assume a branching ratio of one to three for every product pair.

In fact, the energy released by reaction (A.9) absorbed by HOCO radicals can be transferred to the surface before reacting. However, the transfer rate of the chemical energy to the surface is very uncertain. The absence of formic acid $\mathrm{HCOOH}$ and the low abundance of HOCO radicals observed in the experiments of Oba et al. (2010) suggest that $\mathrm{CO}_{2}$ is readily formed from excited HOCO molecules. Therefore, most HOCO radicals continue to react before relaxing to their stable state. To reproduce these experiments, we assume that $99 \%$ of $\mathrm{HOCO}$ radicals are sufficiently excited to form $\mathrm{CO}_{2}$, whilst $1 \%$ of them are stabilized.

Goumans \& Andersson (2010) performed gas-phase O-CO potential energy surface calculations, showing that $\mathrm{O}$ and $\mathrm{CO}$ can form a van der Waals complex, allowing $\mathrm{O}$ atoms to stay bound to $\mathrm{CO}$ for a long time. Following these results, we consider that $\mathrm{O}$ atoms that meet $\mathrm{CO}$ molecules (via direct accretion from gas-phase or surface diffusion) form a loosely bound O...CO complex. The $\mathrm{H}$ atoms that meet these O..CO complexes react via a barrierless reaction to form an excited HO...CO* complex. If the time for energy transfer to the surface is long enough, the complex can yield $\mathrm{OH}+\mathrm{CO}$, or t-HOCO* radical via barrierless reactions. Otherwise, the complex forms the $\mathrm{t}-\mathrm{HOCO}$ radical through quantum tunneling. We consider that $99 \%$ of $\mathrm{HO} . . . \mathrm{CO} *$ complexes continue to react without activation barriers.

Appendix B lists all the reactions involved in the formation of $\mathrm{CO}_{2}$ with their corresponding activation barriers and transmission probabilities.

\section{A.3. Quantum chemical calculations}

The $\mathrm{OH}+\mathrm{H}_{2}$ reaction system has been theoretically studied by Nguyen et al. (2011). These authors have computed the forward and reverse reactions, the imaginary frequency of the transition states, and the rate constants of the eight reactions involving $\mathrm{H}_{2}$, $\mathrm{HD}, \mathrm{D}_{2}, \mathrm{OH}$, and $\mathrm{OD}$ using the semiclassical transition-state theory (SCTST).

The $\mathrm{H}_{2} \mathrm{O}_{2}+\mathrm{H}$ reaction has been studied by Koussa et al. (2006) and Ellingson et al. (2007). However, data for the reactions involving deuterated isotopologues was not available. Therefore, to obtain the data required for the model, quantum chemistry calculations have been conducted with the Gaussian 09 (Frisch et al. 2009) program. For these calculations, the PBE0 (Perdew et al. 1996b,a; Adamo \& Barone 1999) functional and the aug-cc-pVTZ (Dunning Jr 1989; Kendall et al. 1992) basis set were used because this combination produced results that are in good agreement with the experimentally determined values for the process in gas-phase (Klemm et al. 1975, who measured an activation barrier of $4.6 \mathrm{kcal} / \mathrm{mol}=2300 \mathrm{~K}$ ) .

The $\mathrm{CO}+\mathrm{H}$ reaction has been theoretically studied by several authors (Woon 2002; Andersson et al. 2011; Peters et al. 2012). We decided to use the work of Peters et al. (2012) because 
they used the most accurate methodology and obtained a value for the activation energy for the formation of $\mathrm{HCO}$ that best agrees with the gas-phase experiment (Wang et al. 1973). The transmission probabilities of all the reactions producing deuterated formaldehyde and methanol are computed from this reaction and relative rates measured by Nagaoka et al. (2007) and Hidaka et al. (2009) or deduced by TCK12b.

The formation of carbon dioxide includes two reactions having an activation barrier. Quantum calculations of Talbi et al. (2006), Goumans et al. (2008), and Goumans \& Andersson (2010) showed that reaction (11) has an activation barrier of $2500-3000 \mathrm{~K}$, leading to a transmission probability of $5 \times 10^{-23}$ (Goumans \& Andersson 2010; Garrod \& Pauly 2011). Assuming an activation energy of $2500 \mathrm{~K}$ and considering a square barrier, we reproduce this transmission probability with a barrier width of $0.8 \AA$. The transmission probabilities of the reaction pathways involved in reaction (12), and including HOCO radicals, the van der Waals complex HO...CO, and their deuterated isotopologues, were deduced from the potential energy surface computed by Yu et al. (2001). These authors computed the stationary points of the potential energy surface of this reaction using an extrapolated full coupled cluster/complete basis set (FCC/CBS) method. 
V. Taquet et al.: Water ice deuteration: a tracer of the chemical history of protostars

\section{Appendix B: List of grain surface reactions}

Table B.1. List of grain surface chemical reactions considered in this work along with their activation barriers and transmission probabilities.

\begin{tabular}{|c|c|c|c|c|c|c|c|c|}
\hline Reaction & & & & & & & $E_{a}(\mathrm{~K})$ & $P_{\mathrm{r}}$ \\
\hline $\mathrm{H}$ & + & $\mathrm{H}$ & $\rightarrow$ & $(\mathrm{o}) \mathrm{H}_{2}$ & & & 0 & 0.75 \\
\hline $\mathrm{H}$ & + & $\mathrm{H}$ & $\rightarrow$ & (p) $\mathrm{H}_{2}$ & & & 0 & 0.25 \\
\hline $\mathrm{H}$ & + & $\mathrm{D}$ & $\rightarrow$ & HD & & & 0 & 1 \\
\hline D & + & D & $\rightarrow$ & (o) $\mathrm{D}_{2}$ & & & 0 & 0.66 \\
\hline $\mathrm{D}$ & + & D & $\rightarrow$ & (p) $\mathrm{D}_{2}$ & & & 0 & 0.33 \\
\hline $\mathrm{CO}$ & + & $\overline{\mathrm{H}}$ & $\rightarrow$ & $\overline{\mathrm{HCO}}$ & & & 1763 & $1.92(-07)$ \\
\hline $\mathrm{CO}$ & + & $\mathrm{D}$ & $\rightarrow$ & DCO & & & I & $1.92(-08)$ \\
\hline $\mathrm{HCO}$ & + & $\mathrm{H}$ & $\rightarrow$ & $\mathrm{H}_{2} \mathrm{CO}$ & & & 0 & 1 \\
\hline $\mathrm{HCO}$ & + & $\mathrm{D}$ & $\rightarrow$ & HDCO & & & 0 & 1 \\
\hline DCO & + & $\mathrm{H}$ & $\rightarrow$ & HDCO & & & 0 & 1 \\
\hline $\mathrm{DCO}$ & + & D & $\rightarrow$ & $\mathrm{D}_{2} \mathrm{CO}$ & & & 0 & 1 \\
\hline $\mathrm{H}_{2} \mathrm{CO}$ & + & $\mathrm{H}$ & $\rightarrow$ & $\mathrm{CH}_{3} \mathrm{O}$ & & & $T$ & $9.60(-08)$ \\
\hline $\mathrm{H}_{2} \mathrm{CO}$ & + & $\mathrm{D}$ & $\rightarrow$ & $\mathrm{CH}_{2} \mathrm{DO}$ & & & / & $9.60(-09)$ \\
\hline $\mathrm{H}_{2} \mathrm{CO}$ & + & $\mathrm{D}$ & $\rightarrow$ & $\mathrm{HCO}$ & + & HD & / & $9.31(-08)$ \\
\hline $\mathrm{H}_{2} \mathrm{CO}$ & + & $\mathrm{D}$ & $\rightarrow$ & HDCO & + & $\mathrm{H}$ & / & $9.31(-08)$ \\
\hline $\mathrm{HDCO}$ & + & $\mathrm{H}$ & $\rightarrow$ & $\mathrm{CH}_{2} \mathrm{DO}$ & & & / & $1.11(-07)$ \\
\hline $\mathrm{HDCO}$ & + & $\mathrm{D}$ & $\rightarrow$ & $\mathrm{CHD}_{2} \mathrm{O}$ & & & / & $1.11(-07)$ \\
\hline $\mathrm{HDCO}$ & + & $\mathrm{H}$ & $\rightarrow$ & $\mathrm{HCO}$ & + & HD & / & $1.54(-07)$ \\
\hline HDCO & + & $\mathrm{D}$ & $\rightarrow$ & DCO & + & HD & / & $9.31(-08)$ \\
\hline $\mathrm{HDCO}$ & + & $\mathrm{D}$ & $\rightarrow$ & $\mathrm{D}_{2} \mathrm{CO}$ & + & $\mathrm{H}$ & / & $9.31(-08)$ \\
\hline $\mathrm{D}_{2} \mathrm{CO}$ & + & $\mathrm{H}$ & $\rightarrow$ & $\mathrm{CHD}_{2} \mathrm{O}$ & & & / & $1.27(-07)$ \\
\hline $\mathrm{D}_{2} \mathrm{CO}$ & + & D & $\rightarrow$ & $\mathrm{CD}_{3} \mathrm{O}$ & & & I & $1.27(-08)$ \\
\hline $\mathrm{D}_{2} \mathrm{CO}$ & + & $\mathrm{H}$ & $\rightarrow$ & DCO & + & HD & I & $7.30(-08)$ \\
\hline $\mathrm{CH}_{3} \mathrm{O}$ & + & $\mathrm{H}$ & $\rightarrow$ & $\mathrm{CH}_{3} \mathrm{OH}$ & & & 0 & 1 \\
\hline $\mathrm{CH}_{3} \mathrm{O}$ & + & $\mathrm{D}$ & $\rightarrow$ & $\mathrm{CH}_{3} \mathrm{OD}$ & & & 0 & 1 \\
\hline $\mathrm{CH}_{2} \mathrm{OH}$ & + & $\mathrm{H}$ & $\rightarrow$ & $\mathrm{CH}_{3} \mathrm{OH}$ & & & 0 & 1 \\
\hline $\mathrm{CH}_{2} \mathrm{OH}$ & + & D & $\rightarrow$ & $\mathrm{CH}_{2} \mathrm{DOH}$ & & & 0 & 1 \\
\hline $\mathrm{CH}_{2} \mathrm{OD}$ & + & $\mathrm{H}$ & $\rightarrow$ & $\mathrm{CH}_{3} \mathrm{OD}$ & & & 0 & 1 \\
\hline $\mathrm{CH}_{2} \mathrm{OD}$ & + & D & $\rightarrow$ & $\mathrm{CH}_{2} \mathrm{DOD}$ & & & 0 & 1 \\
\hline $\mathrm{CH}_{2} \mathrm{DO}$ & + & $\mathrm{H}$ & $\rightarrow$ & $\mathrm{CH}_{2} \mathrm{DOH}$ & & & 0 & 1 \\
\hline $\mathrm{CH}_{2} \mathrm{DO}$ & + & D & $\rightarrow$ & $\mathrm{CH}_{2} \mathrm{DOD}$ & & & 0 & 1 \\
\hline $\mathrm{CHDOH}$ & + & $\mathrm{H}$ & $\rightarrow$ & $\mathrm{CH}_{2} \mathrm{DOH}$ & & & 0 & 1 \\
\hline $\mathrm{CHDOH}$ & + & $\mathrm{D}$ & $\rightarrow$ & $\mathrm{CHD}_{2} \mathrm{OH}$ & & & 0 & 1 \\
\hline CHDOD & + & $\mathrm{H}$ & $\rightarrow$ & $\mathrm{CH}_{2} \mathrm{DOD}$ & & & 0 & 1 \\
\hline CHDOD & + & $\mathrm{D}$ & $\rightarrow$ & $\mathrm{CHD}_{2} \mathrm{OD}$ & & & 0 & 1 \\
\hline $\mathrm{CHD}_{2} \mathrm{O}$ & + & $\mathrm{H}$ & $\rightarrow$ & $\mathrm{CHD}_{2} \mathrm{OH}$ & & & 0 & 1 \\
\hline $\mathrm{CHD}_{2} \mathrm{O}$ & + & D & $\rightarrow$ & $\mathrm{CHD}_{2} \mathrm{OD}$ & & & 0 & 1 \\
\hline $\mathrm{CD}_{2} \mathrm{OH}$ & + & $\mathrm{H}$ & $\rightarrow$ & $\mathrm{CHD}_{2} \mathrm{OH}$ & & & / & 1 \\
\hline $\mathrm{CD}_{2} \mathrm{OH}$ & + & $\mathrm{D}$ & $\rightarrow$ & $\mathrm{CD}_{3} \mathrm{OH}$ & & & 0 & 1 \\
\hline $\mathrm{CD}_{2} \mathrm{OD}$ & + & $\mathrm{H}$ & $\rightarrow$ & $\mathrm{CHD}_{2} \mathrm{OD}$ & & & 0 & 1 \\
\hline $\mathrm{CD}_{2} \mathrm{OD}$ & + & D & $\rightarrow$ & $\mathrm{CD}_{3} \mathrm{OD}$ & & & 0 & 1 \\
\hline $\mathrm{CD}_{3} \mathrm{O}$ & + & $\mathrm{H}$ & $\rightarrow$ & $\mathrm{CD}_{3} \mathrm{OH}$ & & & 0 & 1 \\
\hline $\mathrm{CD}_{3} \mathrm{O}$ & + & D & $\rightarrow$ & $\mathrm{CD}_{3} \mathrm{OD}$ & & & 0 & 1 \\
\hline $\mathrm{CH}_{3} \mathrm{OH}$ & + & $\mathrm{D}$ & $\rightarrow$ & $\mathrm{CH}_{2} \mathrm{OH}$ & + & HD & T & $2.88(-07)$ \\
\hline $\mathrm{CH}_{2} \mathrm{DOH}$ & + & $\mathrm{D}$ & $\rightarrow$ & $\mathrm{CHDOH}$ & + & HD & / & $1.92(-07)$ \\
\hline $\mathrm{CHD}_{2} \mathrm{OH}$ & + & $\mathrm{D}$ & $\rightarrow$ & $\mathrm{CD}_{2} \mathrm{OH}$ & + & HD & / & $1.50(-07)$ \\
\hline $\mathrm{CH}_{3} \mathrm{OD}$ & + & $\mathrm{D}$ & $\rightarrow$ & $\mathrm{CH}_{2} \mathrm{OD}$ & + & HD & / & $2.88(-07)$ \\
\hline $\mathrm{CH}_{2}$ DOD & + & $\mathrm{D}$ & $\rightarrow$ & CHDOD & + & HD & I & $1.92(-07)$ \\
\hline $\mathrm{CHD}_{2} \mathrm{OD}$ & + & $\mathrm{D}$ & $\rightarrow$ & $\mathrm{CD}_{2} \mathrm{OD}$ & + & HD & 1 & $1.50(-07)$ \\
\hline $\mathrm{O}$ & + & $\mathrm{O}$ & $\rightarrow$ & $\mathrm{O}_{2}$ & & & 0 & 1 \\
\hline $\mathrm{O}_{2}$ & + & $\mathrm{O}$ & $\rightarrow$ & $\mathrm{O}_{3}$ & & & 0 & 1 \\
\hline $\mathrm{O}$ & + & $\mathrm{H}$ & $\rightarrow$ & $\mathrm{OH}$ & & & 0 & 1 \\
\hline $\mathrm{O}$ & + & D & $\rightarrow$ & OD & & & 0 & 1 \\
\hline$\overline{\mathrm{OH}}$ & + & $\mathrm{H}$ & $\rightarrow$ & $\mathrm{H}_{2} \mathrm{O}$ & & & 0 & 1 \\
\hline $\mathrm{OH}$ & + & $\mathrm{D}$ & $\rightarrow$ & HDO & & & 0 & 1 \\
\hline OD & + & $\mathrm{H}$ & $\rightarrow$ & HDO & & & 0 & 1 \\
\hline OD & + & $\mathrm{D}$ & $\rightarrow$ & $\mathrm{D}_{2} \mathrm{O}$ & & & 0 & 1 \\
\hline $\mathrm{OH}$ & + & $\mathrm{OH}$ & $\rightarrow$ & $\mathrm{H}_{2} \mathrm{O}_{2}$ & & & $0(R=0.8)$ & 1 \\
\hline OD & + & $\mathrm{OH}$ & $\rightarrow$ & $\mathrm{HDO}_{2}$ & & & $0(R=0.8)$ & 1 \\
\hline OD & + & OD & $\rightarrow$ & $\mathrm{D}_{2} \mathrm{O}_{2}$ & & & $0(R=0.8)$ & 1 \\
\hline $\mathrm{OH}$ & + & $\mathrm{OH}$ & $\rightarrow$ & $\mathrm{H}_{2} \mathrm{O}$ & + & $\mathrm{O}$ & $0(R=0.2)$ & 1 \\
\hline OD & + & $\mathrm{OH}$ & $\rightarrow$ & HDO & + & $\mathrm{O}$ & $0(R=0.2)$ & 1 \\
\hline OD & + & OD & $\rightarrow$ & $\mathrm{D}_{2} \mathrm{O}$ & + & $\mathrm{O}$ & $0(R=0.2)$ & 1 \\
\hline $\mathrm{OH}$ & + & $\mathrm{H}_{2}$ & $\rightarrow$ & $\mathrm{H}_{2} \mathrm{O}$ & + & $\mathrm{H}$ & 2935 & $4.07(-07)$ \\
\hline
\end{tabular}


Table B.1. continued.

\begin{tabular}{|c|c|c|c|c|c|c|c|c|}
\hline Reaction & & & & & & & $E_{a}(\mathrm{~K})$ & $P_{\mathrm{r}}$ \\
\hline OD & + & $\mathrm{H}_{2}$ & $\rightarrow$ & HDO & + & $\mathrm{H}$ & 2855 & $3.62(-07)$ \\
\hline OD & + & HD & $\rightarrow$ & $\mathrm{D}_{2} \mathrm{O}$ & + & $\mathrm{H}$ & 3051 & $1.00(-09)$ \\
\hline OD & + & $\mathrm{HD}$ & $\rightarrow$ & HDO & + & $\mathrm{D}$ & 3026 & $8.07(-10)$ \\
\hline $\mathrm{OH}$ & + & HD & $\rightarrow$ & $\mathrm{H}_{2} \mathrm{O}$ & + & $\mathrm{D}$ & 2789 & $8.74(-07)$ \\
\hline $\mathrm{OH}$ & + & HD & $\rightarrow$ & HDO & + & $\mathrm{H}$ & 2900 & $2.81(-09)$ \\
\hline $\mathrm{OH}$ & + & $\mathrm{D}_{2}$ & $\rightarrow$ & HDO & + & $\mathrm{D}$ & 2703 & $7.99(-07)$ \\
\hline OD & + & $\mathrm{D}_{2}$ & $\rightarrow$ & $\mathrm{D}_{2} \mathrm{O}$ & + & D & 2870 & $2.26(-09)$ \\
\hline $\mathrm{O}_{2}$ & + & $\mathrm{H}$ & $\rightarrow$ & $\mathrm{HO}_{2}$ & & & 0 & 1 \\
\hline $\mathrm{O}_{2}$ & + & D & $\rightarrow$ & $\mathrm{DO}_{2}$ & & & 0 & 1 \\
\hline $\mathrm{HO}_{2}$ & + & $\mathrm{H}$ & $\rightarrow$ & $\mathrm{H}_{2} \mathrm{O}_{2}$ & & & 0 & 1 \\
\hline $\mathrm{HO}_{2}$ & + & $\mathrm{D}$ & $\rightarrow$ & $\mathrm{HDO}_{2}$ & & & 0 & 1 \\
\hline $\mathrm{DO}_{2}$ & + & $\mathrm{H}$ & $\rightarrow$ & $\mathrm{HDO}_{2}$ & & & 0 & 1 \\
\hline $\mathrm{DO}_{2}$ & + & $\mathrm{D}$ & $\rightarrow$ & $\mathrm{D}_{2} \mathrm{O}_{2}$ & & & 0 & 1 \\
\hline $\mathrm{H}_{2} \mathrm{O}_{2}$ & + & $\mathrm{H}$ & $\rightarrow$ & $\mathrm{H}_{2} \mathrm{O}$ & + & $\mathrm{OH}$ & 2508 & $1.37(-07)$ \\
\hline $\mathrm{H}_{2} \mathrm{O}_{2}$ & + & D & $\rightarrow$ & HDO & + & $\mathrm{OH}$ & 2355 & $5.54(-09)$ \\
\hline $\mathrm{HDO}_{2}$ & + & $\mathrm{H}$ & $\rightarrow$ & HDO & + & $\mathrm{OH}$ & 2523 & $1.23(-07)$ \\
\hline $\mathrm{HDO}_{2}$ & + & $\mathrm{H}$ & $\rightarrow$ & $\mathrm{H}_{2} \mathrm{O}$ & + & OD & 2524 & $1.22(-07)$ \\
\hline $\mathrm{HDO}_{2}$ & + & D & $\rightarrow$ & $\mathrm{D}_{2} \mathrm{O}$ & + & $\mathrm{OH}$ & 2369 & $5.28(-09)$ \\
\hline $\mathrm{HDO}_{2}$ & + & D & $\rightarrow$ & HDO & + & OD & 2367 & $5.29(-09)$ \\
\hline $\mathrm{D}_{2} \mathrm{O}_{2}$ & + & $\mathrm{H}$ & $\rightarrow$ & $\mathrm{D}_{2} \mathrm{O}$ & + & $\mathrm{OH}$ & 2540 & $1.08(-07)$ \\
\hline $\mathrm{D}_{2} \mathrm{O}_{2}$ & + & D & $\rightarrow$ & $\mathrm{D}_{2} \mathrm{O}$ & + & OD & 2384 & $4.28(-09)$ \\
\hline $\mathrm{O}_{3}$ & + & $\mathrm{H}$ & $\rightarrow$ & $\mathrm{O}_{2}$ & + & $\mathrm{OH}$ & 0 & 1 \\
\hline $\mathrm{O}_{3}$ & + & D & $\rightarrow$ & $\mathrm{O}_{2}$ & + & OD & 0 & 1 \\
\hline $\mathrm{N}$ & + & $\mathrm{H}$ & $\rightarrow$ & $\mathrm{NH}$ & & & 0 & 1 \\
\hline $\mathrm{N}$ & + & D & $\rightarrow$ & ND & & & 0 & 1 \\
\hline $\mathrm{NH}$ & + & $\mathrm{H}$ & $\rightarrow$ & $\mathrm{NH}_{2}$ & & & 0 & 1 \\
\hline $\mathrm{NH}$ & + & $\mathrm{D}$ & $\rightarrow$ & NHD & & & 0 & 1 \\
\hline ND & + & $\mathrm{H}$ & $\rightarrow$ & NHD & & & 0 & 1 \\
\hline ND & + & D & $\rightarrow$ & $\mathrm{ND}_{2}$ & & & 0 & 1 \\
\hline $\mathrm{NH}_{2}$ & + & $\mathrm{H}$ & $\rightarrow$ & $\mathrm{NH}_{3}$ & & & 0 & 1 \\
\hline $\mathrm{NH}_{2}$ & + & D & $\rightarrow$ & $\mathrm{NH}_{2} \mathrm{D}$ & & & 0 & 1 \\
\hline NHD & + & $\mathrm{H}$ & $\rightarrow$ & $\mathrm{NH}_{2} \mathrm{D}$ & & & 0 & 1 \\
\hline NHD & + & $\mathrm{D}$ & $\rightarrow$ & $\mathrm{NHD}_{2}$ & & & 0 & 1 \\
\hline $\mathrm{ND}_{2}$ & + & $\mathrm{H}$ & $\rightarrow$ & $\mathrm{NHD}_{2}$ & & & 0 & 1 \\
\hline $\mathrm{ND}_{2}$ & + & $\mathrm{D}$ & $\rightarrow$ & $\mathrm{ND}_{3}$ & & & 0 & 1 \\
\hline $\mathrm{C}$ & + & $\mathrm{H}$ & $\rightarrow$ & $\mathrm{CH}$ & & & 0 & 1 \\
\hline $\mathrm{C}$ & + & D & $\rightarrow$ & $\mathrm{CD}$ & & & 0 & 1 \\
\hline $\mathrm{CH}$ & + & $\mathrm{H}$ & $\rightarrow$ & $\mathrm{CH}_{2}$ & & & 0 & 1 \\
\hline $\mathrm{CH}$ & + & $\mathrm{D}$ & $\rightarrow$ & $\mathrm{CHD}$ & & & 0 & 1 \\
\hline $\mathrm{CD}$ & + & $\mathrm{H}$ & $\rightarrow$ & CHD & & & 0 & 1 \\
\hline $\mathrm{CD}$ & + & $\mathrm{D}$ & $\rightarrow$ & $\mathrm{CD}_{2}$ & & & 0 & 1 \\
\hline $\mathrm{CH}_{2}$ & + & $\mathrm{H}$ & $\rightarrow$ & $\mathrm{CH}_{3}$ & & & 0 & 1 \\
\hline $\mathrm{CH}_{2}$ & + & $\mathrm{D}$ & $\rightarrow$ & $\mathrm{CH}_{2} \mathrm{D}$ & & & 0 & 1 \\
\hline CHD & + & $\mathrm{H}$ & $\rightarrow$ & $\mathrm{CH}_{2} \mathrm{D}$ & & & 0 & 1 \\
\hline CHD & + & D & $\rightarrow$ & $\mathrm{CHD}_{2}$ & & & 0 & 1 \\
\hline $\mathrm{CD}_{2}$ & + & $\mathrm{H}$ & $\rightarrow$ & $\mathrm{CHD}_{2}$ & & & 0 & 1 \\
\hline $\mathrm{CD}_{2}$ & + & $\mathrm{D}$ & $\rightarrow$ & $\mathrm{CD}_{3}$ & & & 0 & 1 \\
\hline $\mathrm{CH}_{3}$ & + & $\mathrm{D}$ & $\rightarrow$ & $\mathrm{CH}_{3} \mathrm{D}$ & & & 0 & 1 \\
\hline $\mathrm{CH}_{2} \mathrm{D}$ & + & $\mathrm{H}$ & $\rightarrow$ & $\mathrm{CH}_{3} \mathrm{D}$ & & & 0 & 1 \\
\hline $\mathrm{CH}_{2} \mathrm{D}$ & + & D & $\rightarrow$ & $\mathrm{CH}_{2} \mathrm{D}_{2}$ & & & 0 & 1 \\
\hline $\mathrm{CHD}_{2}$ & + & $\mathrm{H}$ & $\rightarrow$ & $\mathrm{CH}_{2} \mathrm{D}_{2}$ & & & 0 & 1 \\
\hline $\mathrm{CHD}_{2}$ & + & D & $\rightarrow$ & $\mathrm{CHD}_{3}$ & & & 0 & 1 \\
\hline $\mathrm{CD}_{3}$ & + & $\mathrm{H}$ & $\rightarrow$ & $\mathrm{CHD}_{3}$ & & & 0 & 1 \\
\hline $\mathrm{CD}_{3}$ & + & D & $\rightarrow$ & $\mathrm{CD}_{4}$ & & & 0 & 1 \\
\hline$\overline{\mathrm{HCO}}$ & + & $\mathrm{O}$ & $\rightarrow$ & $\mathrm{CO}_{2}$ & + & $\overline{\mathrm{H}}$ & 0 & 1 \\
\hline DCO & + & $\mathrm{O}$ & $\rightarrow$ & $\mathrm{CO}_{2}$ & + & D & 0 & 1 \\
\hline $\mathrm{CO}$ & + & $\mathrm{O}$ & $\rightarrow$ & $\mathrm{CO}_{2}$ & & & 2500 & $4.80(-23)$ \\
\hline $\mathrm{CO}$ & + & $\mathrm{OH}$ & $\rightarrow$ & $\mathrm{t}-\mathrm{HOCO}$ & & & 285 & $3.50(-03)$ \\
\hline $\mathrm{CO}$ & + & OD & $\rightarrow$ & t-DOCO & & & 121 & $1.63(-01)$ \\
\hline $\mathrm{CO}$ & + & $\mathrm{OH}$ & $\rightarrow$ & $\mathrm{c}-\mathrm{HOCO}$ & & & 2128 & $5.81(-17)$ \\
\hline $\mathrm{CO}$ & + & OD & $\rightarrow$ & c-DOCO & & & 1964 & $9.57(-16)$ \\
\hline t-HOCO* & + & & $\rightarrow$ & $\mathrm{CO}$ & + & $\mathrm{OH}$ & 13050 & $1.63(-10)$ \\
\hline t-HOCO* & + & & $\rightarrow$ & c-HOCO & & & 4114 & 1 \\
\hline t-HOCO & + & $\mathrm{H}$ & $\rightarrow$ & $\mathrm{CO}_{2}$ & + & $\mathrm{H}_{2}$ & 0 & 1 \\
\hline $\mathrm{t}-\mathrm{HOCO}$ & + & $\mathrm{H}$ & $\rightarrow$ & $\mathrm{H}_{2} \mathrm{O}$ & + & $\mathrm{CO}$ & 0 & 1 \\
\hline
\end{tabular}


V. Taquet et al.: Water ice deuteration: a tracer of the chemical history of protostars

Table B.1. continued.

\begin{tabular}{|c|c|c|c|c|c|c|c|c|}
\hline Reaction & & & & & & & $E_{a}(\mathrm{~K})$ & $P_{\mathrm{r}}$ \\
\hline $\mathrm{t}-\mathrm{HOCO}$ & + & $\mathrm{H}$ & $\rightarrow$ & $\mathrm{HCOOH}$ & & & 0 & 1 \\
\hline t-HOCO & + & D & $\rightarrow$ & $\mathrm{CO}_{2}$ & + & HD & 0 & 1 \\
\hline t-HOCO & + & D & $\rightarrow$ & HDO & + & $\mathrm{CO}$ & 0 & 1 \\
\hline t-HOCO & + & $\mathrm{D}$ & $\rightarrow$ & HCOOD & & & 0 & 1 \\
\hline c-HOCO $*$ & + & & $\rightarrow$ & $\mathrm{t}-\mathrm{HOCO}$ & & & 3272 & 1 \\
\hline c-HOCO* & + & & $\rightarrow$ & $\mathrm{CO}_{2}$ & + & $\mathrm{H}$ & 12440 & $2.63(-01)$ \\
\hline c-HOCO & + & & $\rightarrow$ & $\mathrm{t}-\mathrm{HOCO}$ & & & 3272 & $1.19(-17)$ \\
\hline c-HOCO & + & & $\rightarrow$ & $\mathrm{CO}_{2}$ & + & $\mathrm{H}$ & 12440 & $2.94(-21)$ \\
\hline c-HOCO & + & $\mathrm{H}$ & $\rightarrow$ & $\mathrm{CO}_{2}$ & + & $\mathrm{H}_{2}$ & 0 & 1 \\
\hline c-HOCO & + & $\mathrm{H}$ & $\rightarrow$ & $\mathrm{H}_{2} \mathrm{O}$ & + & $\mathrm{CO}$ & 0 & 1 \\
\hline c-HOCO & + & $\mathrm{H}$ & $\rightarrow$ & $\mathrm{HCOOH}$ & & & 0 & 1 \\
\hline c-HOCO & + & D & $\rightarrow$ & $\mathrm{CO}_{2}$ & + & HD & 0 & 1 \\
\hline c-HOCO & + & D & $\rightarrow$ & HDO & + & $\mathrm{CO}$ & 0 & 1 \\
\hline c-HOCO & + & D & $\rightarrow$ & HCOOD & & & 0 & 1 \\
\hline t-DOCO* & + & & $\rightarrow$ & $\mathrm{CO}$ & + & OD & 13220 & $9.27(-02)$ \\
\hline t-DOCO* & + & & $\rightarrow$ & c-DOCO & & & 4238 & 1 \\
\hline t-DOCO & + & $\mathrm{H}$ & $\rightarrow$ & $\mathrm{CO}_{2}$ & + & HD & $0(R=0.33)$ & 1 \\
\hline t-DOCO & + & $\mathrm{H}$ & $\rightarrow$ & HDO & + & $\mathrm{CO}$ & $0(R=0.33)$ & 1 \\
\hline t-DOCO & + & $\mathrm{H}$ & $\rightarrow$ & $\mathrm{DCOOH}$ & & & $0(R=0.33)$ & 1 \\
\hline t-DOCO & + & D & $\rightarrow$ & $\mathrm{CO}_{2}$ & + & $\mathrm{D}_{2}$ & $0(R=0.33)$ & 1 \\
\hline t-DOCO & + & $\mathrm{D}$ & $\rightarrow$ & $\mathrm{D}_{2} \mathrm{O}$ & + & $\mathrm{CO}$ & $0(R=0.33)$ & 1 \\
\hline t-DOCO & + & $\mathrm{D}$ & $\rightarrow$ & DCOOD & & & $0(R=0.33)$ & 1 \\
\hline c-DOCO* & + & & $\rightarrow$ & t-DOCO & & & 3343 & 1 \\
\hline c-DOCO* & + & & $\rightarrow$ & $\mathrm{CO}_{2}$ & + & $\mathrm{D}$ & 13230 & $6.18(-02)$ \\
\hline c-DOCO & + & & $\rightarrow$ & t-DOCO & & & 3343 & $1.51(-22)$ \\
\hline c-DOCO & + & $\mathrm{D}$ & $\rightarrow$ & $\mathrm{CO}_{2}$ & + & $\mathrm{D}_{2}$ & $0(R=0.33)$ & 1 \\
\hline c-DOCO & + & $\mathrm{D}$ & $\rightarrow$ & $\mathrm{D}_{2} \mathrm{O}$ & + & $\mathrm{CO}$ & $0(R=0.33)$ & 1 \\
\hline c-DOCO & + & $\mathrm{D}$ & $\rightarrow$ & DCOOD & & & $0(R=0.33)$ & 1 \\
\hline c-DOCO & + & $\mathrm{H}$ & $\rightarrow$ & $\mathrm{CO}_{2}$ & + & HD & $0(R=0.33)$ & 1 \\
\hline c-DOCO & + & $\mathrm{H}$ & $\rightarrow$ & HDO & + & $\mathrm{CO}$ & $0(R=0.33)$ & 1 \\
\hline c-DOCO & + & $\mathrm{H}$ & $\rightarrow$ & $\mathrm{DCOOH}$ & & & $0(R=0.33)$ & 1 \\
\hline $\mathrm{O}$ & + & $\mathrm{CO}$ & $\rightarrow$ & $\mathrm{O} \ldots \mathrm{CO}$ & & & 0 & 1 \\
\hline O...CO & + & $\mathrm{H}$ & $\rightarrow$ & HO...CO & & & 0 & 1 \\
\hline HO...CO & & & $\rightarrow$ & t-HOCO & & & 775 & $9.46(-09)$ \\
\hline HO...CO & & & $\rightarrow$ & c-HOCO & & & 2618 & $8.17(-21)$ \\
\hline HO...CO* & & & $\rightarrow$ & t-HOCO & & & 775 & 1 \\
\hline $\mathrm{HO} . . . \mathrm{CO} *$ & & & $\rightarrow$ & c-HOCO & & & 2618 & 1 \\
\hline DO...CO & & & $\rightarrow$ & t-DOCO & & & 727 & $2.97(-18)$ \\
\hline DO...CO & & & $\rightarrow$ & c-DOCO & & & 2769 & $1.60(-20)$ \\
\hline DO...CO* & & & $\rightarrow$ & t-DOCO & & & 727 & 1 \\
\hline DO...CO* & & & $\rightarrow$ & c-DOCO & & & 2769 & 1 \\
\hline
\end{tabular}

